\title{
Attenuation mechanisms in fractured fluid-saturated porous rocks: a numerical modelling study
}

\author{
Eva Caspari ${ }^{1 *}$, Mikhail Novikov ${ }^{2,3}$, Vadim Lisitsa ${ }^{2,3}$, Nicolás D. Barbosa ${ }^{1}$, \\ Beatriz Quintal $^{1}$, J. Germán Rubino ${ }^{4}$ and Klaus Holliger ${ }^{1,5}$ \\ ${ }^{1}$ Applied and Environmental Geophysics Group, Institute of Earth Sciences, University of Lausanne, Lausanne, Switzerland, ${ }^{2}$ Novosibirsk \\ State University, Novosibirsk, Russia, ${ }^{3}$ Institute of Petroleum Geology and Geophysics SB RAS, Novosibirsk, Russia, ${ }^{4}$ CONICET, Centro \\ Atómico Bariloche - CNEA, San Carlos de Bariloche, Argentina, and ${ }^{5}$ School of Earth Sciences, Zhejiang University, Hangzhou, China
}

\begin{abstract}
Seismic attenuation mechanisms receive increasing attention for the characterization of fractured formations because of their inherent sensitivity to the hydraulic and elastic properties of the probed media. Attenuation has been successfully inferred from seismic data in the past, but linking these estimates to intrinsic rock physical properties remains challenging. A reason for these difficulties in fluid-saturated fractured porous media is that several mechanisms can cause attenuation and may interfere with each other. These mechanisms notably comprise pressure diffusion phenomena and dynamic effects, such as scattering, as well as Biot's so-called intrinsic attenuation mechanism. Understanding the interplay between these mechanisms is therefore an essential step for estimating fracture properties from seismic measurements. In order to do this, we perform a comparative study involving wave propagation modelling in a transmission set-up based on Biot's low-frequency dynamic equations and numerical upscaling based on Biot's consolidation equations. The former captures all aforementioned attenuation mechanisms and their interference, whereas the latter only accounts for pressure diffusion phenomena. A comparison of the results from both methods therefore allows to distinguish between dynamic and pressure diffusion phenomena and to shed light on their interference. To this end, we consider a range of canonical models with randomly distributed vertical and/or horizontal fractures. We observe that scattering attenuation strongly interferes with pressure diffusion phenomena, since the latter affect the elastic contrasts between fractures and their embedding background. Our results also demonstrate that it is essential to account for amplitude reductions due to transmission losses to allow for an adequate estimation of the intrinsic attenuation of fractured media. The effects of Biot's intrinsic mechanism are rather small for the models considered in this study.
\end{abstract}

Key words: Attenuation, Numerical study, Rock Physics.

\section{INTRODUCTION}

Fractures are of great interest in earth sciences and in civil engineering since they significantly influence the elastic

*E-mail: Eva.Caspari@unil.ch and hydraulic properties of geological formations. Seismic attributes are commonly used to detect and characterize fracture zones (Liu and Martinez 2012). An attribute, which recently gained increased attention for this purpose is seismic attenuation. Seismic attenuation can be broadly divided into intrinsic and apparent attenuation mechanisms. The former 
results in seismic energy loss, whereas the latter redistributes seismic energy. Several attempts have been undertaken to estimate seismic attenuation as well as the respective contributions of intrinsic and apparent attenuation from real data (e.g. Daley, Majer and Peterson 2004; Mangriotis et al. 2013; Alasbali et al. 2016). However, linking these attenuation estimates to intrinsic properties of the rock remains challenging and requires an understanding of the contributions of the different phenomena and their interference.

In fractured fluid saturated porous media, intrinsic and apparent mechanisms which can contribute to wave attenuation and velocity dispersion comprise pressure diffusion phenomena (intrinsic) and dynamic effects such as scattering (apparent) as well as Biot's so-called intrinsic mechanism. In a poroelastic framework, pressure diffusion phenomena, such as wave-induced fluid flow and squirt flow, have been studied numerically (e.g. Rubino et al. 2013, 2014, 2017; Quintal et al. 2014; Vinci, Renner and Steeb 2014; Hunziker et al. 2018) and analytically (e.g. Chapman 2003; Gurevich et al. 2009; Guo et al. 2016), whereas scattering in fractured media is commonly studied in an elastic context (e.g. Saenger, Krüger and Shapiro 2004; Murai 2007; Vlastos et al. 2007) and Biot's intrinsic mechanism only for simple geometries of a single fracture (Wenzlau and Müller 2009; Barbosa et al. 2016). Depending on the frequency regime, the characteristic length scales, and the physical properties of the medium, all of them may contribute to the observed attenuation in a medium containing several fractures. A favourable framework to investigate most of the aforementioned attenuation mechanisms is Biot's theory of poroelasticity (Biot 1941, 1956, 1962a, 1962b), which will be utilized in this study. A mechanism which is not taken into account in this framework is squirt flow, which occurs at the microscopic scale. The considered attenuation mechanisms are described briefly in the following.

In the framework of this study, Biot's intrinsic mechanism refers to the classical Biot relaxation process (Biot 1956, 1962a), which arises at the wavelength scale (Pride 2005) in response to the combined effects of the fluid pressure gradients between the peaks and troughs of the wave and the accelerations induced by the propagating wave. In the remainder of the paper, we refer to this mechanism as Biot global flow. The development of viscous boundary layers at the pore scale due to the dominance of inertial forces over viscous forces is not taken into account as well as the associated relaxation process (Biot 1962b; Johnson, Koplik and Dashen 1987). Thus, the flow in the pore space is governed by Poiseuille flow and we are within the framework of Biot's low-frequency dynamic equations. In the high-frequency regime of these equations, the slow $\mathrm{P}$-wave is a propagating wave.

Scattering attenuation is caused by the finite size of fractures and their elastic contrast with respect to the host rock. Depending on the size of the scatterer with respect to the seismic wavelength, scattering can be divided into three different regimes: Rayleigh, Mie and diffusion scattering. The scenarios considered in this study can be attributed to the Rayleigh scattering regime, where the wavelength is larger than the size of a single fracture. Previous studies (Main, Peacock and Meredith 1990; Vlastos et al. 2007) have shown that the magnitude of scattering depends on multiple factors: the elastic contrast of the scatterer with respect to the host medium, the volume fraction of the scatterers, the path length of the seismic wave and geometrical aspects, such as the size, the orientation and distribution of scatterers.

In a fractured fluid-saturated porous medium pressure diffusion phenomena manifest in two ways (e.g. Gurevich et al. 2009; Müller, Gurevich and Lebedev 2010; Rubino et al. 2013): fracture-to-background wave-induced fluid flow (FB-WIFF) and fracture-to-fracture wave-induced flow (FFWIFF). As in the case of scattering, both are caused by large compressibility contrasts between fractures and their host rocks. These compressibility contrasts result in pressure gradients between fractures and stiff pores of the embedding host rock as well as between intersecting fractures of different orientations upon the incidence of a seismic wave and the relaxation of these pressure gradients is responsible for the energy loss of the seismic wave. For these two mechanisms, the slow P-wave is diffusive. Energy dissipation due to FB-WIFF predominantly occurs in the host rock, whereas FF-WIFF takes place within fractures. Hence, the characteristic time scale for FB-WIFF depends on the hydraulic diffusivity of the host rock (Gurevich et al. 2009). For FF-WIFF, the characteristic time-scale depends on an effective hydraulic diffusivity of the fractures and the host rock (Guo et al. 2016) and the distance between the tip of the fracture and its intersection with an adjacent fracture.

Understanding the interplay between these mechanisms is an essential first step for estimating fracture properties from seismic measurements. To analyse the interference of attenuation phenomena, we follow an approach similar to the ones Gurevich, Zyrianov and Lopatnikov (1997) applied to the scenario of a layered medium and Wenzlau and Müller (2009) applied to a single inclusion or infinite fracture. As in Wenzlau and Müller (2009), our study is based on wave propagation simulations of Biot's low-frequency dynamic equations (Biot 1956, 1962a). Although there have been several finite 

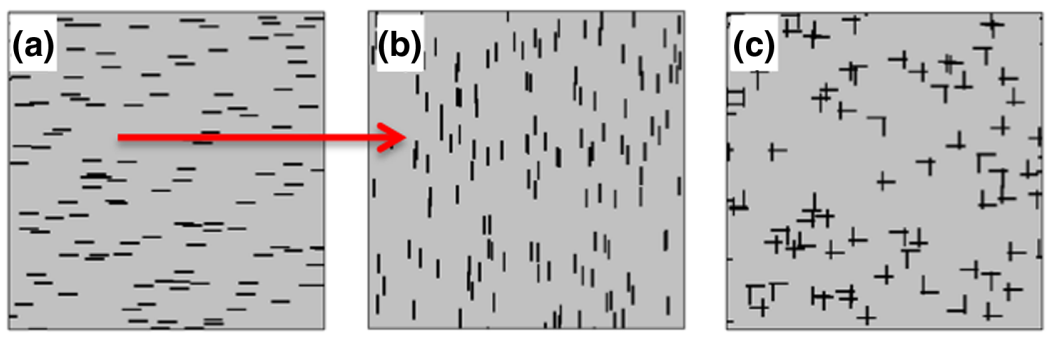

Figure 1 Stochastic realization of the fracture models for (a) parallel, (b) perpendicular and (c) intersecting fractures. The red arrow denotes the direction of wave propagation. difference schemes developed over the last years to perform such simulations, there is a lack of studies for more complex media. One reason is certainly that wave propagation studies of diffusion processes are computationally expensive. Another reason is that the resulting attenuation estimates also tend to be difficult to interpret when various mechanisms interfere with each other. Here, we utilize our finite difference code, (Novikov et al. 2017a, b) which is based on the implementation of Masson, Pride and Nihei (2006). We consider numerical models with randomly distributed horizontal and vertical fractures in a $2 \mathrm{D}$ fluid-saturated porous medium and run simulations over a relatively broad frequency range to capture dynamic and diffusion attenuation processes. To guide the interpretation of the attenuation estimates from the wave propagation results, which capture all three processes, we utilize a complementary numerical upscaling technique based on the quasi-static poroelastic equations (Biot 1941). Such upscaling methods have become popular for estimating attenuation caused by pressure diffusion effects (e.g. Masson and Pride 2007; Rubino, Ravazzoli and Santos 2009; Wenzlau and Müller 2009; Quintal et al. 2011; Jänicke, Quintal and Steeb 2015). Thus, a comparison of the results from both methods allows to distinguish pressure diffusion phenomena and dynamic effects, as has been shown in our preliminary study for fractured media (Novikov et al. 2017a, b). The aim of this study is to analyse the effects of various attenuation mechanisms in a fractured medium on the seismic waveforms and to shed a light on their interference and impact on the attenuation estimates.

The paper is structured as follows. We first present the numerical fracture models considered in this study. Then, we briefly describe the numerical methods and their set-ups for the simulations. The modelling results are shown next and the effects of attenuation on the recorded waveforms are discussed. Finally, we compare the attenuation estimates of both methods. In order to do this, we also have to take transmission losses into account, which occur in the wave propagation simulations. Finally, we discuss the interplay between these attenuation mechanisms.

\section{FRACTURED MEDIUM}

The 2D numerical models considered in this study consist of a water-saturated porous medium containing uniform random distributions of fractures. To explore the effects of the various attenuation mechanisms on the seismic wavefields, we consider three scenarios: a fracture set parallel to wave propagation, one perpendicular to wave propagation and one with intersecting fractures (Fig. 1). The size of a single fracture is $30 \mathrm{~mm}$ by $4 \mathrm{~mm}$ and the fracture density, defined as the area covered by fractures per unit area, is $6.25 \%$ for all models. Although the relative large aspect ratio is not realistic for natural fractures, it still captures the main features of a fractured medium, while making the wave propagation simulations feasible. The fractures themselves are considered to be part of the poroelastic continuum with very high porosity, low matrix stiffness moduli and a tortuosity close to 1 . A tortuosity of 1 represents the case of freely flowing fluid between two plates, a slightly higher value accounts in our case for the porous nature of the fractures. The host rock is modelled as a stiff low-porosity medium. To limit the frequency range in the wave propagation simulations, we study various effects of the diffusion mechanisms and their interference with scattering attenuation by varying the permeability and thus the hydraulic diffusivity of the fractures and host rock over several orders of magnitude for a variety of hypothetical scenarios. All rock and fluid properties are listed in Table 1.

\section{METHODS}

We consider two complementary numerical methods to distinguish between dynamic and diffusion-based attenuation mechanisms. On the one hand, we employ wave propagation simulations based on a finite difference solution of Biot's dynamic equations (Biot 1956, 1962a) and, on the other hand, a numerical upscaling technique based on the quasi-static poroelastic equations (Biot 1941). The former accounts for both types of attenuation mechanisms, whereas the latter takes only diffusion processes into consideration. Thus, a 
Table 1 Rock and fluid properties

\begin{tabular}{lllll}
\hline Property & Symbol & Unit & Background & Fracture \\
\hline Bulk modulus of solid grains & $K_{s}$ & $\mathrm{GPa}$ & 37 & 37 \\
Shear modulus of solid grains & $\mu_{s}$ & $\mathrm{GPa}$ & 44 & 44 \\
Density of solid grains & $\rho_{s}$ & $\mathrm{~kg} / \mathrm{m}^{3}$ & 2650 & 2650 \\
Bulk modulus of fluid & $K_{f}$ & $\mathrm{GPa}$ & 2.25 & 2.25 \\
Fluid viscosity & $\eta$ & $\mathrm{Pa} \cdot \mathrm{s}$ & $10^{-3}$ & $10^{-3}$ \\
Density of fluid & $\rho_{f}$ & $\mathrm{~kg} / \mathrm{m}^{3}$ & 1090 & 1090 \\
Shear modulus of dry frame & $\mu$ & $\mathrm{GPa}$ & 31 & 0.01 \\
Bulk modulus of dry frame & $K_{b}$ & - & 0.1 & 0.02 \\
Porosity & $\phi$ & - & 1.83 & 0.9 \\
Tortuosity & $\tau$ & $\mathrm{GPa}$ & 1.1 \\
\hline Property & $\mathrm{Unit}$ & $\mathrm{Perpendicular}$ & Parallel & Intersecting \\
\hline Background permeability & $\mathrm{m}^{2}$ & $10^{-10}-10^{-17}$ & $10^{-17}$ & $10^{-17}$ \\
Fracture permeability & $\mathrm{m}^{2}$ & $10^{-9}$ & $10^{-14}$ & $10^{-9}-10^{-14}$ \\
\hline
\end{tabular}

comparison of the results from both methods allows for distinguishing between pressure diffusion phenomena and dynamic effects.

\subsection{Poroelastic wave propagation modelling}

In the framework of Biot's theory (Biot 1956, 1962a), a fluidsaturated porous medium consists of a porous elastic solid saturated with a viscous and compressible fluid. In such a medium, S- and P-waves as well as can exist. The fast S- and $\mathrm{P}$-waves correspond to classical body waves in an isotropic elastic or viscoelastic medium, whereas the slow P-wave develops due to the fluid in the pore space. A seismic wave travelling through such a fluid-saturated porous medium creates a relative fluid-solid displacement, which is dominated by viscous forces in the low-frequency regime, resulting in a diffusive slow P-wave (pressure diffusion wave), and by inertial forces in the high-frequency regime, resulting in a propagating slow $\mathrm{P}$-wave. This relative fluid movement in the pore space can be described by

$-\nabla p=\eta \mathbf{Y} * \mathbf{q}-\rho_{f} \frac{\partial \mathbf{v}}{\partial t}$

where $\mathbf{v}, \mathbf{q}=\phi\left(\mathbf{v}_{\mathrm{f}}-\mathbf{v}\right), \mathbf{v}_{\mathrm{f}}$ are the solid phase, the relative fluidsolid phase and fluid phase displacement velocities, respectively, and $p, \rho_{f}$ and $\eta$ denote the fluid pressure, density and viscosity, respectively. A problem of this formulation for numerical simulations of wave propagation in the time domain is the time convolution-like non-local pseudo-differential operator $\mathrm{Y} * \mathrm{q}$, where $\mathrm{Y}$ denotes the viscodynamic operator. The reason is that it requires the storage of the wavefield at all-time instants. There are several ways to overcome this drawback (Carcione 1996; Masson et al. 2006; Carcione and Davide 2009; Carcione, Morency and Santos 2010; Masson and Pride 2010). Here, we utilize the approach of Masson et al. (2006), which yields a low-frequency generalized Darcy's law

$-\nabla p=\rho_{f} \frac{\tau}{\phi} \frac{\partial \mathbf{q}}{\partial t}+\rho_{f} \frac{\partial \mathbf{v}}{\partial t}+\frac{\eta}{k_{0}} \mathbf{q}$.

Following Carcione et al. (2010), we retained all the coefficients which can be experimentally measured, that is, tortuosity $\tau$, porosity $\phi$, and fluid density $\rho_{f}$. This low-frequency approximation does not account for effects due to the development of viscous boundary layers at the pore scale, which would require the concept of a dynamic permeability to be accounted for (Johnson et al. 1987). Therefore, the corresponding relaxation process is not captured in our study and the flow in the pore space is governed by Poiseuille flow. For a detailed discussion of this topic, we refer to Masson et al. (2006) and Pride (2005). However, Biot's classical relaxation mechanism (Biot global flow) due to the combined effects of fluid pressure gradients between peaks and troughs of the wave and the accelerations induced by the propagating wave is taken into account.

The final dynamic equations for an isotropic, homogeneous poroelastic medium in the space-time domain are given by

$\nabla \cdot \boldsymbol{\sigma}=\rho_{f} \frac{\partial \mathbf{q}}{\partial t}+\rho \frac{\partial \mathbf{v}}{\partial t}$

$-\nabla p=\rho_{f} \frac{\tau}{\phi} \frac{\partial \mathbf{q}}{\partial t}+\rho_{f} \frac{\partial \mathbf{v}}{\partial t}+\frac{\eta}{k_{0}} \mathbf{q}$ 


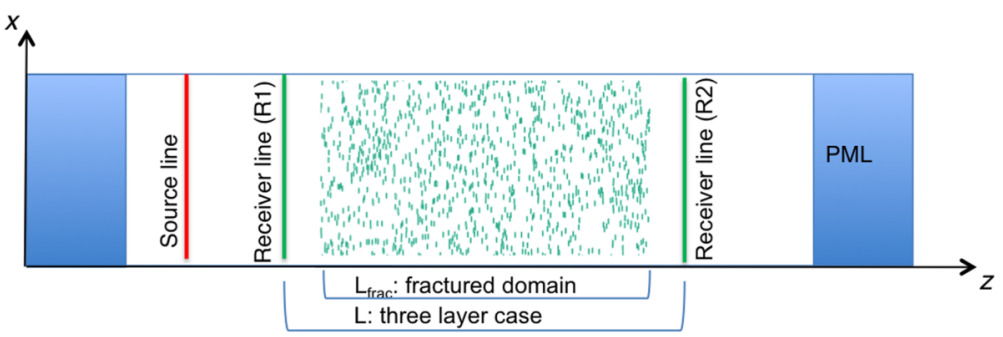

Figure 2 Schematic illustration of the computational domain. The red line corresponds to the source positions, green lines represent the receiver positions. At the left and right boundary perfectly matched layers (PML) are implemented.

where $\sigma$ denotes the total stress tensor and equation (4) is the total balance of forces acting on the fluid-solid system. The density of the saturated rock is given by $\rho=(1-\phi) \rho_{s}+\phi \rho_{f}$, where $\phi$ is the porosity and $\rho_{s}$ is the solid density. The constitutive equations are

$\frac{\partial \boldsymbol{\sigma}}{\partial t}=\left(\lambda_{u} \nabla \cdot \mathbf{v}+\alpha M \nabla \cdot \mathbf{q}\right) \mathbf{I}+\mu\left[\nabla \mathbf{v}+\left(\nabla \mathbf{v}^{\mathrm{T}}\right)\right]$,

$-\frac{\partial p}{\partial t}=M(\alpha \nabla \cdot \mathbf{v}+\nabla \cdot \mathbf{q})$,

where $\lambda_{u}$ is the Lamé parameter of the undrained rock and $\mu$ is the shear modulus. The Biot-Willis coefficient $\alpha$ and fluid storage modulus $M$ are defined as

$\alpha=1-K_{d} / K_{s}$

$M=B K_{u} / \alpha$,

$B=\frac{1 / K_{d}-1 / K_{s}}{1 / K_{d}-1 / K_{s}+\phi\left(1 / K_{f}-1 / K_{s}\right)}$,

$K_{u}=\frac{K_{d}}{1-B\left(1-K_{d} / K_{s}\right)}$,

where $K_{d}, K_{u}$, and $K_{s}$ are the bulk moduli of the drained and undrained rock and of the solid constituents, respectively. Details of the implemented finite difference approximation are given in Appendix A.

\subsubsection{Numerical set-up}

The wave propagation simulations are performed for a $2 \mathrm{D}$ transmission set-up shown in Fig. 2. The fractured medium is embedded in a homogeneous background medium, which has the same properties as the host rock (Table 1). A plane P-wave is generated by a line of point sources in the homogeneous part of the model and the wavefields are recorded at two receiver lines, one located before and one after the fractured medium. To obtain the frequency-dependent energy dissipation in such a transmission set-up over a relative broad frequency range, we employ band-limited Ricker wavelets with a compact time history at different central frequencies. The thickness of the fractured layer varies with the central frequency of the wavelet so that the layer thickness corresponds to two to five wavelengths. The minimum layer thickness is at least 20 times the correlation length of the heterogeneity (fracture) distribution and the height of the model domain is $1 \mathrm{~m}$. The central frequencies and layer thicknesses are listed in Table 2. To avoid a reduction of fracture concentrations at the top and bottom of the 2D modelling domain, the geometry of the fractured medium is periodic along these boundaries and periodic boundary conditions are applied. At the left and right boundaries, complex frequency-shifted perfectly matched layers (PML) (Berenger 1994; Drossaert and Giannopoulos 2007) are implemented to prevent artificial reflections. The model is discretized on a regular grid with a grid spacing of $1 \mathrm{~mm}$, which results in four grid cells within the fractures and is sufficient to capture the diffusion processes appropriately.

The recorded traces at each receiver line are stacked to a single trace. The incoming signal of the plane P-wave (receiver line 1) and the transmitted P-wave signal (receiver line 2) are separated from the other arrivals, for example coda waves by a tapered time window. For each central frequency of these recordings, the phase velocity and attenuation are estimated by the spectral ratio technique (Gurevich and Pevzner 2015). The details are given in Appendix B.

\subsection{Quasi-static numerical upscaling}

We apply the numerical upscaling scheme of Rubino et al. (2016) for anisotropic 2D media in the frequency domain, which is based on a finite element solution of Biot's consolidation equations (Biot 1941). By neglecting all inertia-related terms and transforming equations (3-4) into the spacefrequency domain, we obtain the following coupled system of the total stress equilibrium and Darcy's law expressed in displacements

$\nabla \cdot \sigma=0$ 
Table 2 Set up parameters for the transmission experiment

\begin{tabular}{llllll}
\hline Frequency $(\mathrm{kHz})$ & Domain length $(\mathrm{m})$ & Source line $(\mathrm{m})$ & Receiver line 1 $(\mathrm{m})$ & Receiver line 2 $(\mathrm{m})$ & Fractured domain $(\mathrm{m})$ \\
\hline 0.5 & 110 & 15 & 25 & 95 & $45-85$ \\
1 & 60 & 10 & 15 & 50 & $25-45$ \\
2 & 35 & 7.5 & 10 & 27.5 & $15-25$ \\
3 & 26.7 & 6.67 & 8.34 & 20.03 & $11.68-18.36$ \\
4 & 22.5 & 6.25 & 7.5 & 16.25 & $10-15$ \\
5 & 20 & 6 & 7 & 14 & $9-13$ \\
6 & 18.4 & 5.84 & 6.68 & 12.56 & $8.36-11.72$ \\
7 & 17.2 & 5.72 & 6.44 & 1.48 & $7.88-10.76$ \\
8 & 16.3 & 5.63 & 6.26 & 10.67 & $7.52-10.04$ \\
9 & 15.6 & 5.56 & 6.12 & 9.04 & $7.24-9.48$ \\
10 & 15 & 5.5 & 6 & 9.5 & $7-9$ \\
\hline
\end{tabular}

$-\nabla p=i \omega \frac{\eta}{\kappa_{o}} \mathbf{w}$

The relative fluid-solid displacement is given by $\mathbf{w}=$ $\phi(\mathbf{U}-\mathbf{u})$, where $\mathbf{U}$ and $\mathbf{u}$ are the macroscopic fluid and solid displacements, respectively. The constitutive equations (5-6) of the poroelastic medium in space-frequency domain are

$\boldsymbol{\sigma}=\left[\lambda_{u} \nabla \cdot \mathbf{u}+\alpha M \nabla \cdot \mathbf{w}\right] \mathbf{I}+\mu\left[\nabla \mathbf{u}+(\nabla \mathbf{u})^{T}\right]$

$-p=M(\alpha \nabla \cdot \mathbf{u}+\nabla \cdot \mathbf{w})$.

\subsubsection{Numerical set-up}

Three oscillatory relaxation tests, comprising two compressional tests and one shear test, are applied to a $1 \mathrm{~m}$ by $1 \mathrm{~m}$ square sample of the fractured domain utilized in the wave propagation modelling. The model is discretized on a regular mesh and the same grid spacing of $1 \mathrm{~mm}$ as in the wave propagation simulations is used. The two compressional tests are performed by applying a time-harmonic vertical displacement at the top and bottom of the sample for test one and a horizontal displacement at the lateral boundaries for test two. Along the remaining boundaries the sample is fixed. The third test is a simple shear test. In all tests, the sample is hydraulically sealed so that the fluid is not allowed to flow in or out. The mathematical expressions of the boundary conditions are given in Appendix C.

From the spatially averaged complex-valued stress and strain fields of the three tests, we obtain a frequencydependent complex valued 2D stiffness matrix in Voigt notation by applying a least square procedure (Rubino et al. 2016). The following relation holds

$$
\left(\begin{array}{c}
\left\langle\sigma_{11}(\omega)\right\rangle \\
\left\langle\sigma_{22}(\omega)\right\rangle \\
\left\langle\sigma_{12}(\omega)\right\rangle
\end{array}\right)=\left(\begin{array}{lll}
C_{11} & C_{12} & C_{16} \\
C_{12} & C_{22} & C_{26} \\
C_{16} & C_{26} & C_{66}
\end{array}\right)\left(\begin{array}{c}
\left\langle\epsilon_{11}(\omega)\right\rangle \\
\left\langle\epsilon_{22}(\omega)\right\rangle \\
\left\langle 2 \epsilon_{12}(\omega)\right\rangle
\end{array}\right),
$$

where the stiffness coefficients $C_{i j}$ describe an effective anisotropic viscoelastic behaviour of the fractured poroelastic medium, provided that the sub-sample has the size of a representative elementary volume (REV). The $1 \mathrm{~m}$ by $1 \mathrm{~m}$ samples are assumed to be sufficiently close to the REV size. From the complex-valued coefficients, the phase velocity and attenuation of $\mathrm{P}$ - and $\mathrm{S}$-waves as function of frequency and incidence angle are determined by solving a plane wave problem for a viscoelastic medium. The procedure is described in detail in Rubino et al. (2016). Here, we only focus on the P-wave velocity and attenuation for horizontal incidence, which is given by:

$V_{p}(\omega, \theta)=\frac{\omega}{\Re\left(l_{p}(\omega, \theta)\right)}$.

$Q_{p}^{-1}(\omega, \theta)=\frac{\Im\left(l_{p}(\omega, \theta)^{2}\right)}{\Re\left(l_{p}(\omega, \theta)^{2}\right)}$,

where $l_{p}$ is the effective $\mathrm{P}$-wave number of the fractured medium. Setting the incidence angle as $\theta=0$, we obtain the P-wave attenuation and velocity of a horizontally incident wave, which corresponds to the set-up of the transmission experiment.

\section{MODELLING RESULTS}

To illustrate how fluid-saturated fractures affect the waveforms in a transmission experiment, traces recorded at the two receiver lines for different central frequencies are shown in the following for the three scenarios of a fractured medium at selected permeabilities and frequencies. The permeabilities, 
which in turn determine the hydraulic diffusivities of the host rock and fractures, are chosen so that the fracture models capture FB WIFF in the perpendicular case and FF-WIFF in the intersecting case while none of the two mechanisms plays a significant role in the parallel case. The corresponding attenuation and phase velocities from the oscillatory test, which capture the diffusion mechanisms (FB-WIFF and FF-WIFF), are shown as functions of frequency alongside the waveforms. The central frequencies are selected so that waveforms correspond to a regime with and without scattering. The recorded traces correspond to the velocity of the solid phase, since we are interested in the attenuation of the fast P-wave. A propagating slow P-wave cannot be clearly identified for this wavefield. Furthermore, at the first receiver line, apart from the incoming signal, a reflection from the fractured zone is recorded for all cases and frequencies. This in turn indicates that not only the former mentioned attenuation mechanisms will lead to an amplitude reduction, but that some of the reduction is caused by transmission losses between the two receiver lines. In the numerical set-up of this study, transmission losses comprise the reflections of the fast and slow P-wave and transmitted slow P-waves at the interfaces between the embedding background and fractured domain.

\subsection{Perpendicular case}

The results of the transmission experiment are shown in Fig. 3(a) for three permeabilities which correspond to the cases of the low-frequency regime $\left(\kappa_{b}=10^{-10} \mathrm{~m}^{2}\right)$, the highfrequency regime $\left(\kappa_{b}=10^{-17} \mathrm{~m}^{2}\right)$ and the regime of FB-WIFF $\left(\kappa_{b}=10^{-13} \mathrm{~m}^{2}\right)$ with respect to the considered diffusion mechanism. At a central frequency of $500 \mathrm{~Hz}$, the stiffening effect of the rock from the low-frequency regime $\left(\kappa_{b}=10^{-10} \mathrm{~m}^{2}\right)$, where the induced fluid pressure gradients have time to equilibrate during the half cycle of the wave, to the high-frequency regime $\left(\kappa_{b}=10^{-17} \mathrm{~m}^{2}\right)$, where the induced pressure within the fractures reduces their compliance, can be clearly observed by the faster arrival of the transmitted signal in the latter case. The corresponding effective velocities of the oscillatory test are shown in Fig. 3(c). In both, low- and high-frequency regime, the attenuation due to FB-WIFF is close to zero as evidenced in Fig. 3(b). Nevertheless, there is a slight amplitude difference between the two cases (Fig. 3a, R2). One explanation might be the occurrence of a propagating slow $\mathrm{P}$-wave at the permeability of $\kappa_{b}=10^{-10} \mathrm{~m}^{2}$ and associated losses due to conversion of fast waves to slow P-waves at the interfaces between the fractures and the matrix. Another reason is the difference in transmission losses for the two media. An indication of the latter is the slightly smaller amplitude of the reflected wave (a)
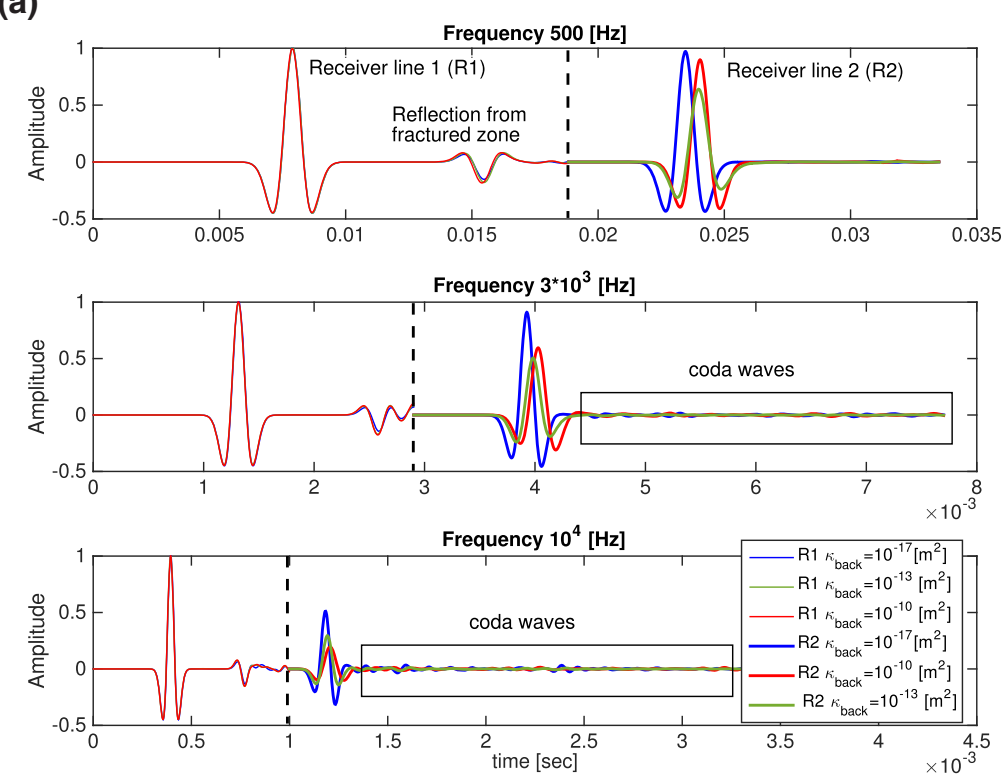

(b)

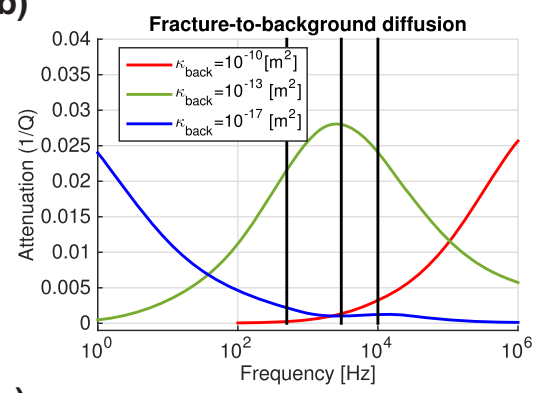

(c)

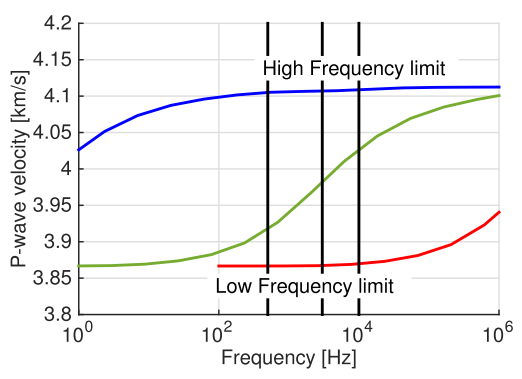

Figure 3 (a) Transmission experiment for the perpendicular case: Recorded averaged waveforms at receiver line 1 and receiver line 2 for three different central frequencies and permeabilities. The black dashed line separates the two recordings. Oscillatory test: (b) resulting P-wave attenuation and (c) phase velocities as function of frequency for the same permeabilities. The black lines correspond to the central frequencies of the signals shown in (a). 
for $\kappa_{b}=10^{-17} \mathrm{~m}^{2}$ compared to the case with $\kappa_{b}=10^{-10} \mathrm{~m}^{2}$. For the third permeability $\left(\kappa_{b}=10^{-13} \mathrm{~m}^{2}\right)$, noticeable attenuation and dispersion occurs due to FB-WIFF at the considered frequency of $500 \mathrm{~Hz}$ as indicated by the oscillatory test. The attenuation is reflected by the strong amplitude reduction of the transmitted signal and the corresponding positive dispersion by a change of shape of the signal pulse, which undergoes a phase shift compared to the two non-dispersive signals at receiver 2 and the incoming signal at receiver 1 (Fig. $3 \mathrm{a}, 500 \mathrm{~Hz}$ ).

At the two higher central frequencies of 3 and $10 \mathrm{kHz}$ scattering starts to affect the response (Fig. 3a). At these frequencies coda waves are recorded for all three permeabilities and marked by the black box in Fig. 3(a). The transmitted signals recorded at receiver 2 experience a phase shift opposite in sign compared to those associated with the diffusion processes. The reason is that Rayleigh scattering is governed by negative dispersion. The amplitude loss is more significant for the two cases with relatively high permeabilities and lower effective velocities compared to the one for the low-permeability medium, for which the effective velocity is high and the contrast between fractures and host rock is reduced due to the stiffening of the fractures (Fig. 3c).

\subsection{Intersecting case}

As for the perpendicular fracture case, the transmission results are shown for three permeabilities and frequencies (Fig. 4a).
As expected from the oscillatory tests (Fig. 4b and c), the effects of attenuation and dispersion due to pressure diffusion on the seismic waveforms are smaller than in the perpendicular fracture scenario. The main reason is that the amount of perpendicular fractures, which contribute to the stiffening effect from low to high frequencies, is reduced by $50 \%$. The other $50 \%$ are parallel fractures, since we kept the fracture density constant for all three scenarios. Parallel fractures do not experience a noticeable compression during the passage of the seismic wave and thus do not experience significant induced fluid pressures. For the considered frequency regime, the pressure diffusion takes place between connected fractures (FF-WIFF), where the parallel fractures act as fluid storage volume and not the host rock as in the previous scenario. At a central frequency of $1000 \mathrm{~Hz}$, we observe small differences for the three permeabilities with respect to the arrival times of the transmitted signals and their amplitudes, which are in agreement with the pressure diffusion mechanism (Fig. 4b and c), that is the signal corresponding to the case with the highest permeability arrives later than the signal corresponding to the case with a low permeability. The shape of the transmitted signal at a $1000 \mathrm{~Hz}$ for a permeability of $\kappa_{\text {frac }}=10^{-13} \mathrm{~m}^{2}$, at which FF-WIFF causes the strongest attenuation (Fig. 4b) experiences a slight phase shift in sign opposite to the phase shift of the signals for the other two permeabilities. The former is indicative of the diffusion process (positive dispersion), whereas the latter indicates scattering (negative dispersion). (a)
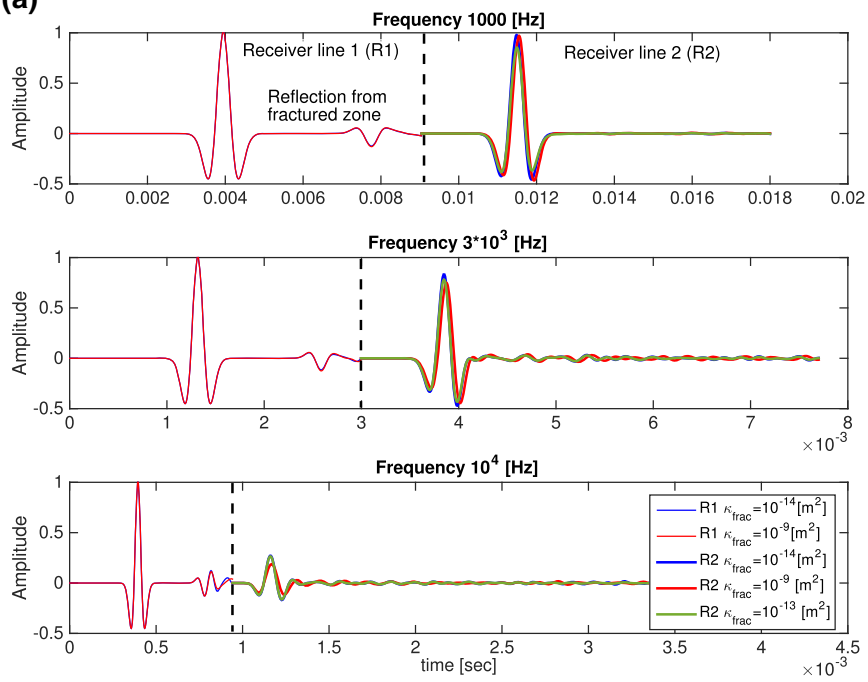

(b)

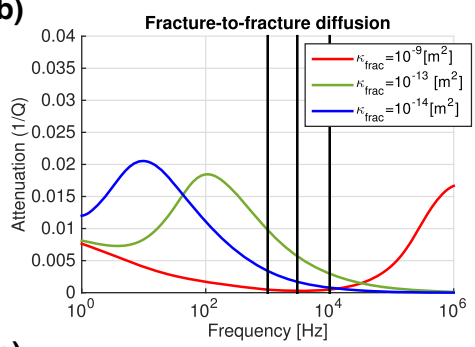

(c)

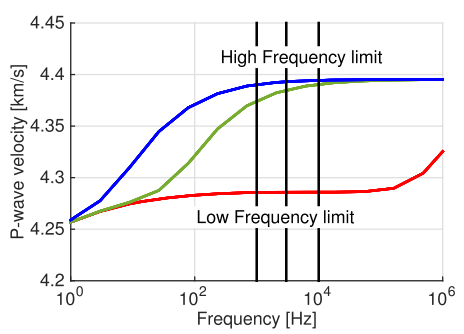

Figure 4 (a) Transmission experiment for the intersecting case: recorded averaged waveforms at receiver line 1 and receiver line 2 for three different central frequencies and selected permeabilities. The black dashed line separates the two recordings. (b) Oscillatory test: resulting P-wave attenuation and (c) phase velocities as function of frequency for the same selected permeabilities. The black lines correspond to the central frequencies of the signals shown in (a). 
(a)
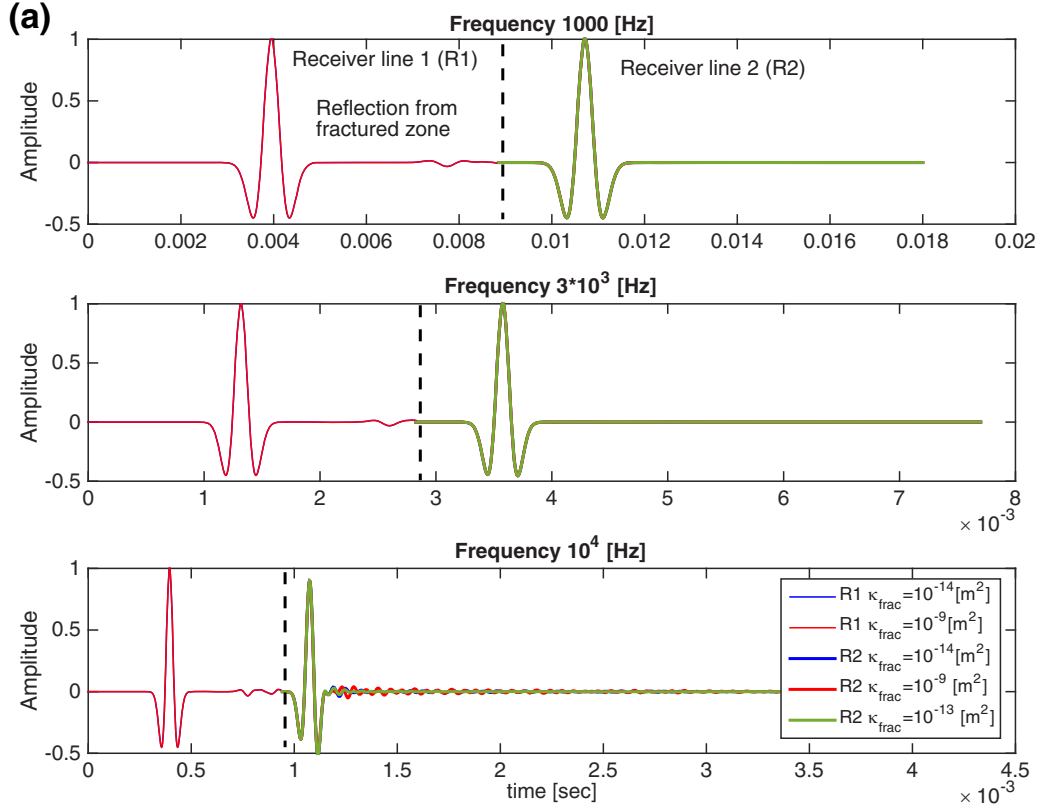

(b)

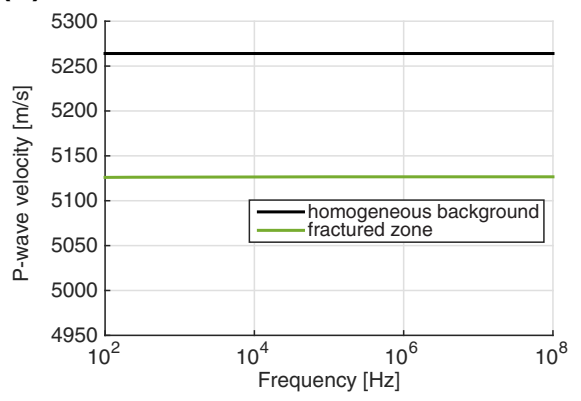

Figure 5 (a) Transmission experiment for the parallel case: Recorded averaged waveforms at receiver line 1 and receiver line 2 for three different central frequencies and permeabilities. The black dashed line separates the two recordings. (b) Effective phase velocity of the fractured zone obtained from the oscillatory test and the phase velocity of the surrounding homogeneous medium.

A closer inspection of the traces confirms that scattering affects the seismic signal at this frequency, as indicated by the small amplitude coda waves (visible for the red and blue curves in Fig. 4a). At the two higher central frequencies, scattering starts to dominate the response of the transmitted signals and correspondingly all signals show similar phase shifts.

\subsection{Parallel case}

The transmission experiments for the two lower central frequencies do not show noticeable amplitude or wavelet changes (Fig. 5a) for the different permeabilities. Since all fractures are parallel with respect to the direction of wave propagation, pressure diffusion effects are very small. Furthermore, due to the choice of the low permeability host medium as in the case for intersecting fractures, FB-WIFF falls outside of the considered frequency range. Such a low background permeability also prevents a slow propagating P-wave in the host rock, although the fracture properties would theoretically support slow waves. The fractures still sufficiently lower the impedance of the medium to create a reflection at the interface between the homogenous and fractured zone (Fig. 5a), which is recorded at receiver line 1. In addition to the effective velocity of the fractured domain obtained from the oscillatory test, we plot the velocity of the homogeneous medium surrounding the fractured zone (Fig. 5b), which confirms a non-negligible velocity contrast between the two zones. Furthermore, scattering can be clearly observed for the highest displayed central frequency.

\section{COMPARISON OF ATTENUATION AND VELOCITY ESTIMATES}

In the transmission experiment, the wave travels between the two receiver lines through a three-layer medium (homogeneous - fractured - homogeneous) of length $L$ (Fig. 2), whereas the oscillatory test accounts only for the fractured domain of length $L_{\text {frac }}$. In order to compare the resulting attenuation and velocity estimations for the two complementary numerical methods, we have to either correct the wave propagation results for the additional travel distance in the homogeneous medium or the results of the oscillatory test for a three-layer case.

\subsection{Effective velocities}

Since we know the velocities in the homogeneous embedding medium, it is straightforward to correct the velocity estimates 
(a)

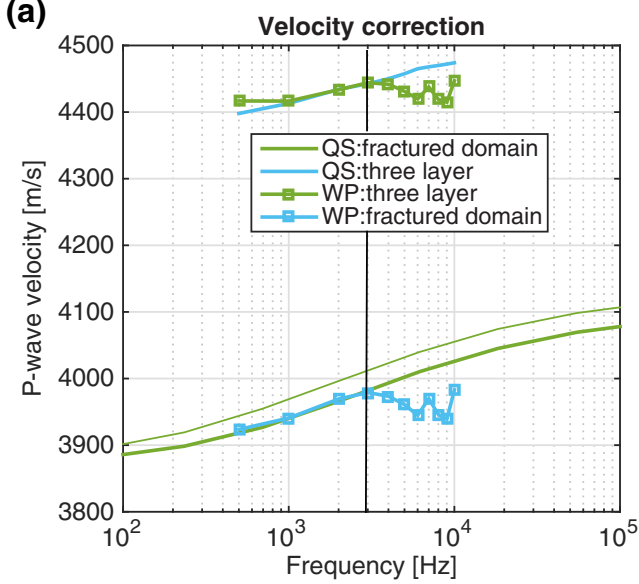

(b)

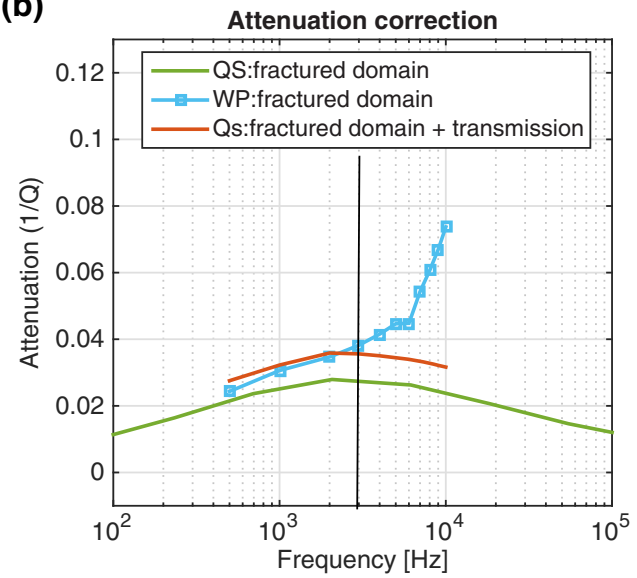

Figure 6 (a) Effective velocities for the fractured domain and the three-layer case and their corresponding corrections (blue lines) for the transmission experiment (WP) and oscillatory tests (QS). The two solid green lines correspond to two sub-samples of the fractured medium. The black line indicates the onset of scattering. (b) Attenuation estimates for the fractured domain from the transmission experiment (WP) and oscillatory tests (QS) with and without transmission losses.

of the two numerical methods. The effective P-wave velocity $V_{p}^{*(\text { three })}$ for the three-layer case and fractured domain $V_{p}^{*(\text { frac })}$ are given by:

$V_{p}^{*(\text { three })}=\frac{L}{t_{\text {total }}}, \quad t_{\text {total }}=\frac{L_{\text {frac }}}{V_{p}^{\text {visco }}}+\frac{L-L_{\text {frac }}}{V_{p}^{\text {bomo }}}$,

$V_{p}^{*(\text { frac })}=\frac{L_{\text {frac }}}{t_{\text {frac }}}, \quad t_{\text {frac }}=\frac{L_{\text {frac }}}{V_{p}^{\text {visco }}}$,

where $t_{\text {total }}$ and $L$ are the total travel time and distance between the two receiver lines, respectively and $L_{\text {frac }}, t_{\text {frac }}, V_{p}^{\text {visco }}$ and $V_{p}^{\text {bomo }}$ denote the length, travel time and velocity of the

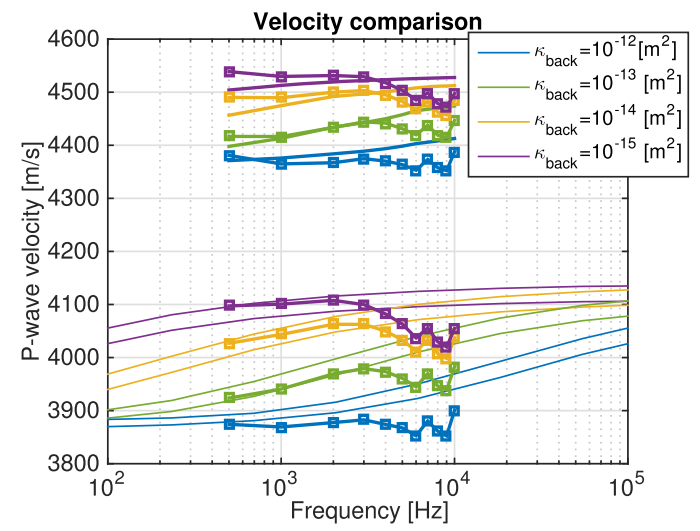

Figure 7 Effective velocities for the fractured domain (bottom set of curves) and the three-layer case (top set of curves) and their corresponding corrections for four different permeabilities. The solid lines correspond to the oscillatory test and the squares to the wave propagation results. fractured domain and velocity of the homogeneous domain, respectively. Figure 6(a) displays the resulting velocities for the fractured domain and three-layer case for the perpendicular scenario for which fracture-to-background wave-induced fluid flow (FB-WIFF) $\left(\kappa_{b}=10^{-13} \mathrm{~m}^{2}\right)$ occurs. The velocities show a very good agreement between the two methods until scattering starts to dominate the wave propagation response (indicated by the black line in Fig. 6a). The two velocity estimates of the oscillatory test correspond to two sub-samples of the fractured domain. The velocity difference can be attributed to slight variabilities of fracture density of the subsamples and to the fact that at a domain size of $1 \times 1 \mathrm{~m}$ the representative elementary volume (REV) size has not yet been fully reached. However, the perpendicular scenario, comparing the velocities for different permeabilities to the wave propagation results, indicates that the overall agreement is still good (Fig. 7). The $\mathrm{REV}$ issue is most severe for the perpendicular case, for which the fractures have the largest impact on the effective velocities due to pressure diffusion (Caspari et al. 2016).

\subsection{Attenuation and transmission losses}

Contrary to the velocities, applying corresponding corrections to the attenuation is more difficult due to the additional amplitude reduction caused by transmission losses. To estimate transmission losses, we perform a $2 \mathrm{D}$ plane-wave amplitude analysis for an anisotropic viscoelastic layer embedded in an isotropic elastic medium. We use an elastic approximation for the embedding homogeneous poroelastic medium, since the attenuation due to Biot global flow is 

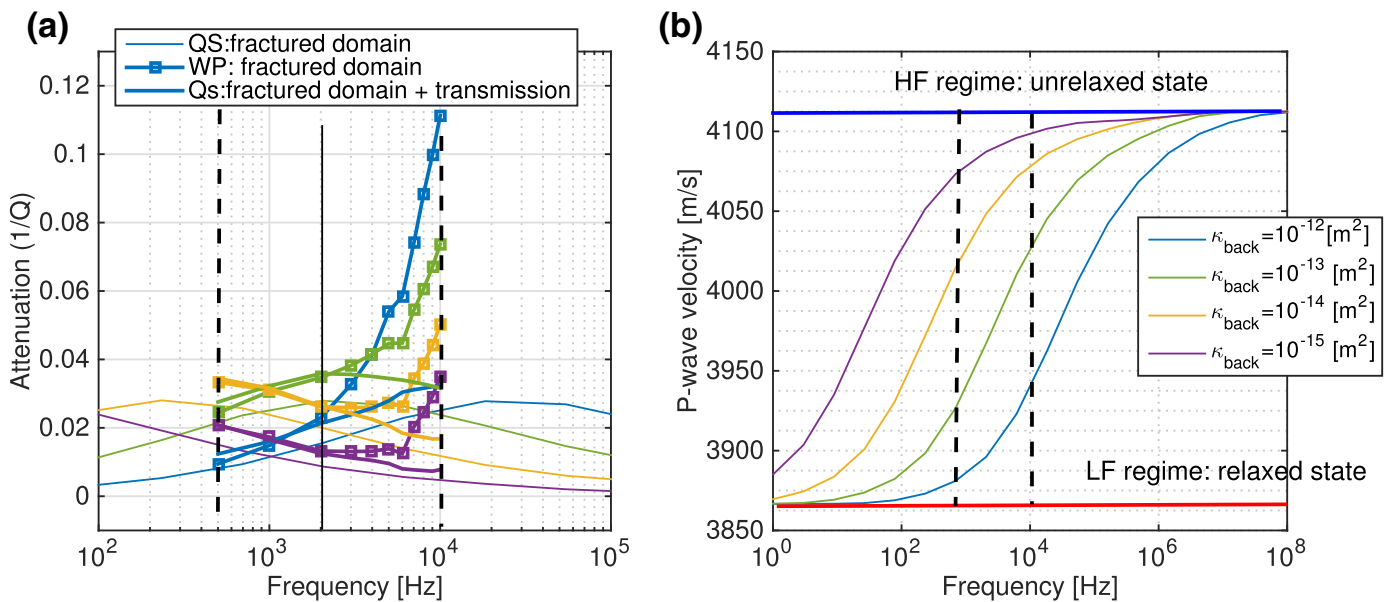

Figure 8 Perpendicular case: (a) Attenuation estimates for four different permeabilities. The black solid line indicates the onset of scattering. (b) Corresponding P-wave velocity estimate from the oscillatory test. The black dashed lines indicate the frequency range of the wave propagation simulations.

negligibly small. Furthermore, transmission losses due to reflected and transmitted slow $\mathrm{P}$-waves are not considered at the interface between the embedding and fractured domain. The viscoelastic layer properties are given by the $C_{i j}$ coefficients of the oscillatory test. The details are given in Appendix D. Once the amplitudes of all generated wave modes are known, the displacement fields of the normally incident and transmitted waves can be obtained. Assuming that the decay in the P-wave solid displacement is due to the viscoelastic layer with frequency-dependent attenuation and dispersion equal to that of the fractured medium, the effective P-wave number of the three-layer case as function of the homogeneous layer properties and the transmission losses is given by

$l_{p}^{e f f}=\frac{-i l_{p} L+\ln \left(A_{t}\right)}{-i L}$

where $l_{p}$ and $A_{t}$ are the P-wave number of the homogeneous domain and the amplitude of the transmitted wave, respectively. From the effective P-wave number, the attenuation can then be computed as follows:

$Q_{p}^{-1}=\frac{\Im\left(\left(l_{p}^{e f f}\right)^{2}\right)}{\Re\left(\left(l_{p}^{e f f}\right)^{2}\right)}$.

By interchanging $L$ with $L_{\text {frac }}$ in equation (20), the effective P-wave number for the fractured domain can be retrieved. Figure 6(b) shows the attenuation estimates for the fractured domain of the oscillatory test with and without transmission losses compared to the wave propagation results. As for the velocities, we observe an excellent agreement till scattering attenuation starts to play a role. In the following, we focus on the attenuation in the fractured domain and show the wave propagation results, the quasistatic results and the transmission-corrected quasi-static results.

\subsection{Perpendicular case: FB-WIFF}

Figure $8(\mathrm{a})$ shows the attenuation for four different permeabilities of the host rock at which FB-WIFF occurs. At frequencies below $2 \mathrm{kHz}$, this pressure diffusion mechanism is predominant as evidenced by the good agreement between the oscillatory test and transmission experiment. This also confirms that the wave propagation modelling adequately captures the diffusion process in the host rock. Above $2 \mathrm{kHz}$, scattering attenuation starts to affect the response of the transmission experiment and we observe a difference in the attenuation estimates with respect to the oscillatory test. Correspondingly, pressure diffusion effects become more difficult to observe at high frequencies. Nevertheless, the magnitude of the scattering attenuation strongly varies between the four cases, since the effective compliance of a fracture in a fluid-saturated porous medium is influenced by the diffusion process. Depending on the frequency and hydraulic diffusivity, the latter varying with the permeability of the host rock, induced pressure gradients either relax during the wave cycle (low-frequency regime) and fractures behave more compressible, are unrelaxed (high-frequency regime) and the fluid pressure stiffens the fractures, or resume an intermediate state. The effective velocities (Fig. $8 \mathrm{~b}$ ) illustrate the 
(a)

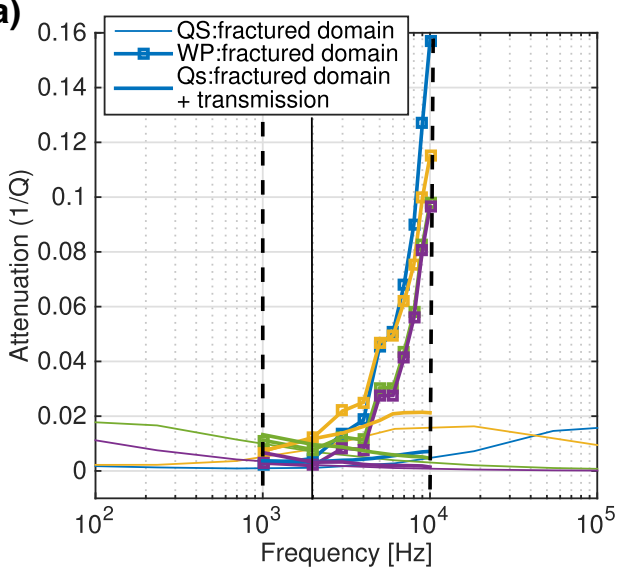

(b)

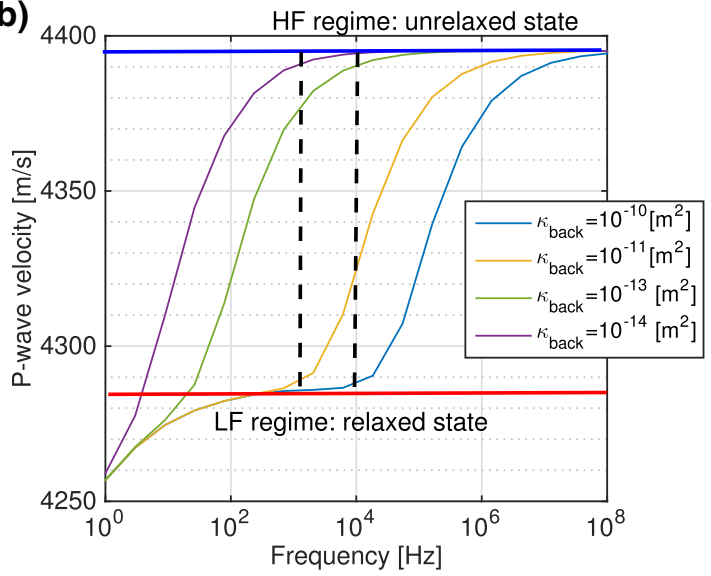

Figure 9 Intersecting case: (a) Attenuation estimates for four different permeabilities. The black solid line indicates the onset of scattering. (b) Corresponding P-wave velocity estimate from the oscillatory test. The black dashed lines indicate the frequency range of the wave propagation simulations.

stiffening of the medium from low to high frequencies and are an indicator of the fracture stiffness. The largest contrast between fractures and their host rock occurs in the relaxed state and thus we expect the strongest scattering attenuation for this scenario. For all other scenarios the scattering attenuation will be smaller. The attenuation curves in Fig. 8(a) correspond to the intermediate states. As expected, we do indeed observe a clear increase in attenuation with hydraulic diffusivity (permeability), since we are approaching the relaxed state (Fig. 8b).

\subsection{Intersecting case: FF-WIFF}

Figure 9(a) shows the attenuation for four different permeabilities of the fractured medium at which FF-WIFF occurs. As in the perpendicular scenario below $2 \mathrm{kHz}$, the results of the oscillatory test and transmission experiment are in good agreement, and, thus at these frequencies FF-WIFF dominates, whereas at higher frequencies strong scattering attenuation controls the response of the transmission experiment. Accounting for transmission losses improves the agreement between the two numerical methods in some cases indicating that they are not negligible (Fig. 10). However, for the two lowest permeabilities, we overestimate the transmission losses. This might be due to REV issues in the oscillatory test or uncertainties in the estimation of quite small attenuation values for the transmission experiment. Nevertheless, the difference in the magnitude of scattering attenuation (Fig. 9a) can be attributed to fracture-to-fracture pressure diffusion, which determines the effective compliance of the fractures and thus their contrast with respect to the host rock. The process is the same as for the perpendicular scenario, except that the diffusion process occurs within the fractures and not in the host medium. The increase of the effective velocities (Fig. 9b) from low to high frequencies are again an indicator of the change in fracture compliance. The overall dispersion for FF-WIFF compared to FB-WIFF is smaller since, as discussed in Section 4.2, only fractures perpendicular to the wave propagation experience an induced fluid pressure upon the incidence of the seismic wave, whereas fractures parallel to the wave propagation direction act as the storage volume for this diffusion process. This is also reflected in the smaller attenuation magnitude caused by pressure diffusion for intersecting fractures compared to perpendicular fractures.

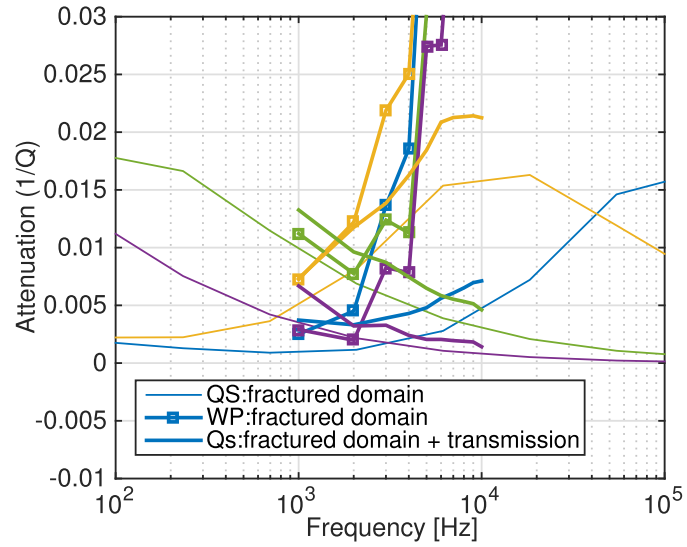

Figure 10 Zoom of Fig. 9(a): Comparison of attenuation estimates from the transmission experiment and the oscillatory test with and without correction for transmission losses. 
(a)

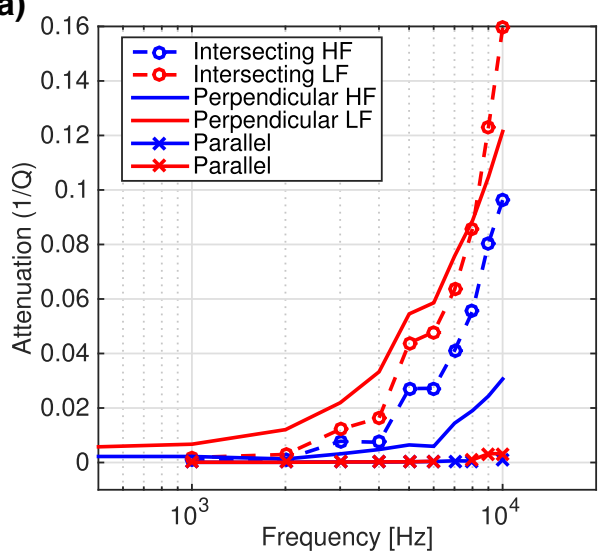

(b)

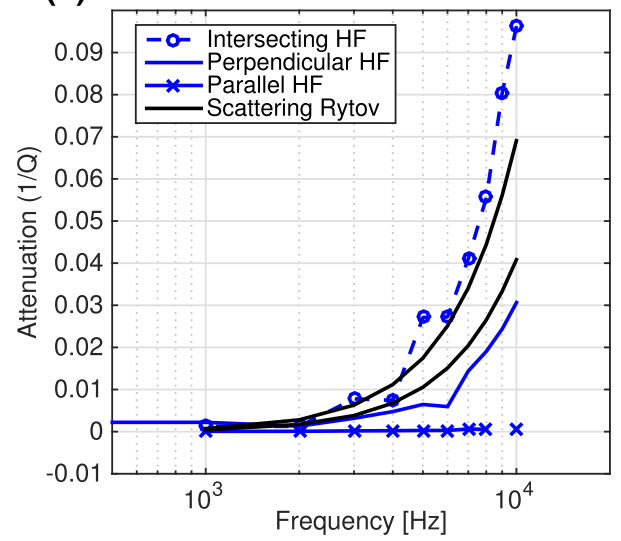

Figure 11 Scattering: (a) Comparison of scattering attenuation in the low-frequency (LF) and high-frequency (HF) regimes for the perpendicular, intersecting and parallel scenarios. (b) Comparison of scattering attenuation in the HF regime with an analytical solution of Rytov et al. (1988).

\subsection{Scattering attenuation}

As discussed in the previous sections, scattering attenuation affects the response of the transmission experiments above a frequency of $2 \mathrm{kHz}$ for the perpendicular and the intersecting scenarios. The magnitude of the scattering attenuation for each type of fractured medium varies considerably depending on the effective compliance of the fractures, which in turn is determined by the prevailing pressure diffusion process. The largest difference in terms of fracture compliance occurs between the low- and high-frequency regimes (Barbosa et al. 2016), at which attenuation due to pressure diffusion processes does not occur. A comparison of scattering attenuation estimates in these two regimes is shown in Fig. 11(a) for all three fractured media and for permeabilities corresponding to low- and high-frequency regimes, respectively. As expected,

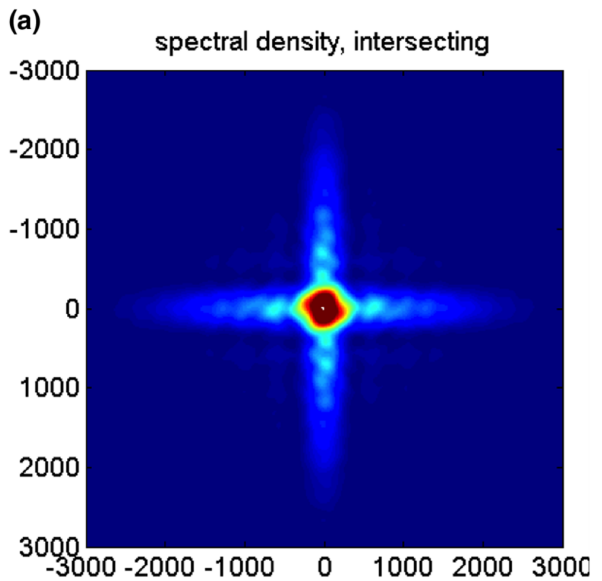

the attenuation in the low-frequency regimes is larger than in the high-frequency regimes for all scenarios. Despite the contrast between fractures and the host rock, scattering attenuation is influenced by the geometry of the fracture arrangement. The geometrical influence can be illustrated by the classical scattering theory of Rytov, Kravtsov and Tatarskii (1988) and is detailed in Appendix E. This theory accounts for geometrical factors, such as, in our case, the distance between fractures as well as their orientation and connectivity in terms of the spectral density of the slowness fluctuations of the medium. The spectral densities for the perpendicular and intersecting cases are shown in Fig. 12. Their intensities are indicative for the scattering strength. We observe clear differences in the shape as well as their intensity. The resulting attenuation estimates are shown in Fig. 11(b). They capture the main trend and confirm that the difference

(b)

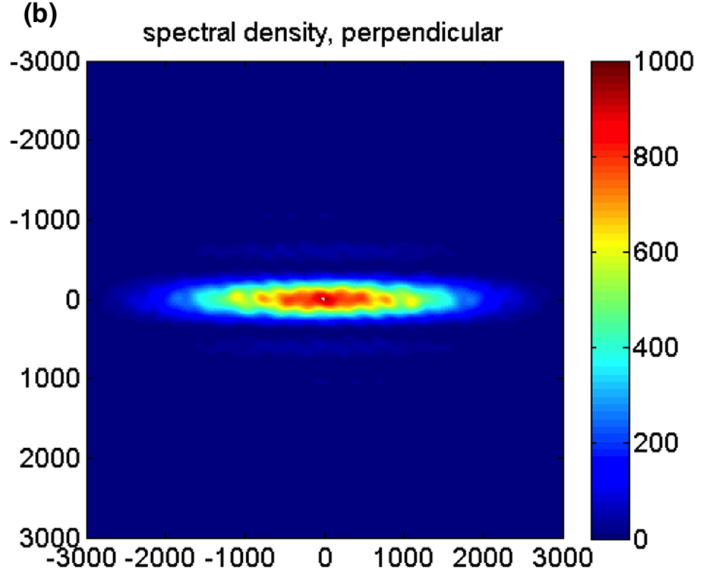

Figure 12 (a) Spectral density for the intersecting (b) and perpendicular scenario. The colour code is indicative of the scattering strength of the medium. 
(a)

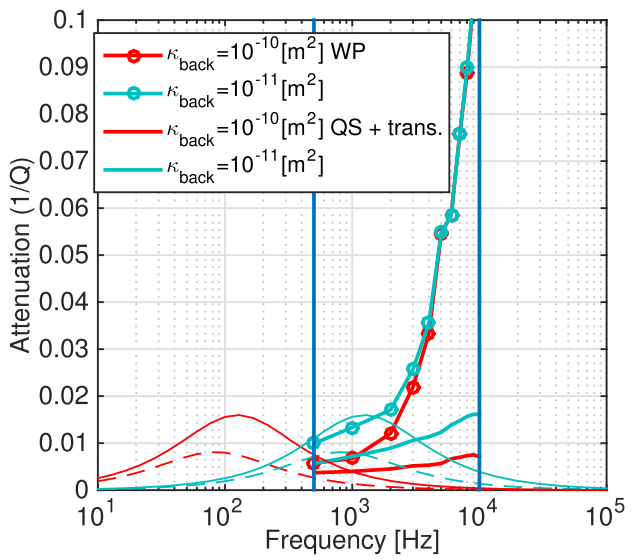

(b)

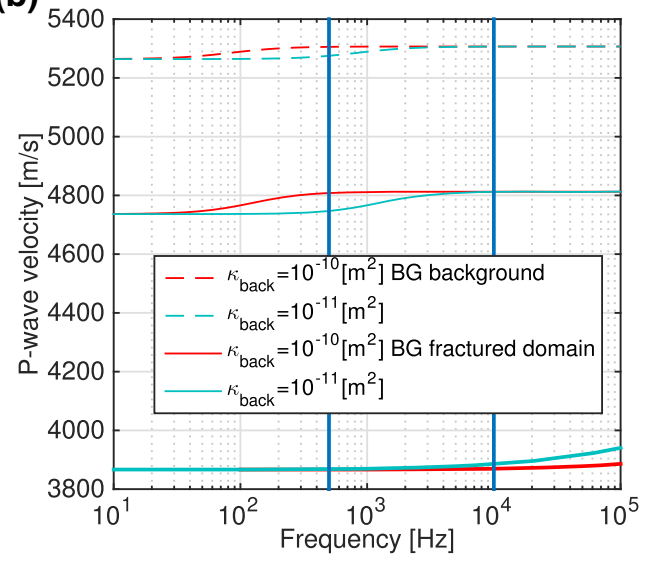

Figure 13 Perpendicular scenario at $\kappa_{b}=10^{-10}$ and $10^{-11} \mathrm{~m}^{2}$ : (a) Attenuation estimates for the transmission experiment (WP), the oscillatory test including transmission losses (QS + trans.), Biot global flow of the host rock properties (BG background) and of the effective fractured medium properties (BG fractured domain) and (b) corresponding velocities. Note that the legends of the subplots are valid for both of them.

in scattering between the perpendicular and intersecting cases is governed by the geometry of the scatterers. However, the absolute values are not precisely reproduced, since the theory is based on the assumption of an acoustic medium.

\subsection{Biot global flow}

So far, we have focused on diffusion processes and their interaction with scattering attenuation. However, the transmission experiments also account for Biot global flow. For the perpendicular scenario, this mechanism occurs in the host rock at permeabilities of $10^{-10}-10^{-12} \mathrm{~m}^{2}$. The corresponding attenuation for the transmission experiment is plotted in Fig. 13(a) for $\kappa_{b}=10^{-10}$ and $10^{-11} \mathrm{~m}^{2}$. At frequencies below $3 \mathrm{kHz}$, we observe clear differences between these two cases. A comparison to attenuation estimates from the oscillatory test including transmission losses (Fig. 13a, Qs + trans) cannot fully explain this difference. Moreover, the difference in transmission losses for the cases is expected to be small as indicated by the corresponding velocities (Fig. 13b). This suggests that Biot global flow might play a role. To analyse this further, we estimate the attenuation and velocities corresponding to Biot global flow for the host rock properties (Fig. 13, BG background) and for the effective properties of the fractured medium (Fig. 13, BG fracture). In the latter case, the effective permeability and tortuosity are approximated by a simple harmonic average of the fracture and host rock properties and the dry properties are obtained from the oscillatory test. Note that the estimation of Biot global flow for the fractured domain is a first-order approximation, since it does not take the anisotropic nature of the fractured medium into account (Milani et al. 2016). Nevertheless, the resulting attenuations of the two analytical Biot global flow estimates for the two background premeabilities $\left(\kappa_{b}=10^{-10}\right.$ and $\kappa_{b}=10^{-11} \mathrm{~m}^{2}$ ) show differences of similar magnitude as the transmission experiment at low frequencies. This indicates that at $\kappa_{b}=10^{-11} \mathrm{~m}^{2}$ Biot global flow is likely to contribute. At higher frequencies, the differences between the two cases in the transmission experiment disappear and the response seems to be solely governed by scattering. Although there are observable differences in the attenuation estimates from the oscillatory test, they seem to play not a role in the transmission experiments. The reason may be that the slow $\mathrm{P}$-wave is no longer a diffusive wave but a propagating wave. In this regime, the quasi-static equations of the oscillatory test are strictly not valid. For the intersecting and parallel scenarios, the medium properties within the fractures would theoretically support Biot global flow, but we do not observe any corresponding evidence in the resulting attenuation estimates. This is not surprising since the wavelength of the slow P-wave only becomes comparable to the aperture and length of the fractures for frequencies above $10 \mathrm{kHz}$ which are not considered in this study and for which we would expect similar effects as those observed for a single infinite fracture embedded in a poroelastic medium (Barbosa et al. 2016).

\section{CONCLUSIONS}

In this study, we applied two complementary numerical methods, wave propagation simulations in form of transmission experiments and a numerical upscaling scheme based on oscillatory relaxation tests, to analyse attenuation mechanisms 
and their interference in fluid-saturated fractured media. Very good agreement between attenuation estimates from the transmission experiments and the corresponding estimates from the oscillatory relaxation tests at low frequencies is observed for most scenarios after correcting for transmission losses. This verifies that the attenuation behaviour caused by pressure diffusion phenomena at low frequencies is properly captured by both numerical methods. We also observe that it is crucial to account for the travel path of the wave and amplitude reductions due to transmission losses, if one wants to obtain the intrinsic attenuation of the fractured medium.

While attenuation is governed by pressure diffusion at low frequencies, attenuation due to scattering increases rapidly at higher frequencies, which makes the observation of WIFF effects more difficult. The strong scattering attenuation is the result of the chosen fracture apertures, which lead to aspect ratios that are not representative for natural fractures, but make the wave propagation simulations feasible. Nevertheless, the results clearly indicate that scattering attenuation is strongly affected by pressure diffusion phenomena, since they determine the effective compliance of the fractures and thus the contrast between fractures and host rock. Another important aspect which controls the magnitude of scattering is the geometrical arrangement of fractures. Although the fracture density is the same for all three types of the fractured media considered in this study, the scattering magnitudes differ. As expected, scattering is lowest for fractures parallel to the direction of wave propagation. The highest scattering attenuation occurs for intersecting fractures, even though the compliance contrast in this case is smaller than for the perpendicular arrangement, as the cross-section of the scatter is the determining factor here.

The effects of Biot global flow are rather small for the considered models and can be only clearly identified for the two lowest host rock permeabilities in the perpendicular scenario. Interestingly, scattering attenuation at higher frequencies does not seem to be affected when the slow P-wave is propagating.

\section{ACKNOWLEDGEMENTS}

This work has been completed within the Swiss Competence Center on Energy Research - Supply of Electricity, with the support of Innosuisse. V. Lisitsa and M. Novikov are thankful to the Russian Foundation for Basic Research grants no. 17-35-50043, 18-05-00031, 18-01-00579, 18-55-20002 and
16-05-00800 for financial support of the research. Simulations of seismic wave propagation were performed on clusters of the Siberian Supercomputer Center. J. Germán Rubino gratefully acknowledges an extended visit to the University of Lausanne financed by the Fondation Herbette.

\section{REFERENCES}

Alasbali A., Pevzner R., Tertyshnikov K., Bóna A. and Gurevich B. 2016. Estimation of seismic attenuation and prediction of VTI anisotropy parameters from VSP and log data: a case study from the Middle East. Arabian Journal of Geosciences 9, 485.

Barbosa N.D., Rubino J.G., Caspari E., Milani M. and Holliger K. 2016. Fluid pressure diffusion effects on the seismic reflectivity of a single fracture. The Journal of the Acoustical Society of America 140, 2554-2570.

Berenger J.P. 1994. A perfectly matched layer for the absorption of electromagnetic waves. The Journal of Computational Physics 114, 185-200.

Biot M.A. 1956. Theory of propagation of elastic waves in a fluidsaturated porous solid. I. Low-frequency range. Journal of the Acoustical Society of America 28, 168-178.

Biot M.A. 1962a. Mechanics of deformation and acoustic propagation in porous media. Journal of Applied Physics 33, 14821498.

Biot M.A. 1962b. Generalized theory of acoustic propagation in porous dissipative media. The Journal of the Acoustical Society of America 34, 1254-1264.

Biot M.A. 1941. General theory for three-dimensional consolidation. Journal of Applied Physics 12, 155-164.

Carcione J.M. 1996. Wave propagation in anisotropic, saturated porous media: plane wave theory and numerical simulation. The Journal of the Acoustical Society of America 99, 26552666.

Carcione J.M. and Davide G. 2009. Theory and numerical simulation of fluid-pressure diffusion in anisotropic porous media. Geophysics 74, N31-N39.

Carcione J.M., Morency C. and Santos J.E. 2010. Computational poroelasticity - A review. Geophysics 75, 75A229-75A243.

Caspari E., Milani M., Rubino G.J., Müller T.M., Quintal B. and Holliger K. 2016. Numerical upscaling of frequency-dependent P- and S-wave moduli in fractured porous media. Geophysical Prospecting 64, 1166-1179.

Chapman M. 2003. Frequency-dependent anisotropy due to mesoscale fractures in the presence of equant porosity. Geophysical Prospecting 51, 369-379.

Daley T.M., Majer E.L. and Peterson J.E. 2004. Cross-well seismic imaging in a contaminated basalt aquifer. Geophysics 69, 16-24.

Drossaert F.H. and Giannopoulos A. 2007. Complex frequency shifted convolution PML for FDTD modelling of elastic waves. Wave motion 44, 593-604.

Dutta N.C. and Odé H. 1983. Seismic reflections from a gas-water contact. Geophysics 48, 148-162. 
Guo J., Rubino G.J., Glubokovskikh S. and Gurevich B. 2016. Effects of fracture intersections on seismic dispersion: theoretical predictions versus numerical simulations. Geophysical Prospecting 65, 1264-1276.

Gurevich B., Brajanovski M., Galvin R.J., Müller T.M. and TomsStewart J. 2009. P-wave dispersion and attenuation in fractured and porous reservoirs-poroelasticity approach. Geophysical Prospecting 57, 225-237.

Gurevich B. and Pevzner R. 2015. How frequency dependency of $\mathrm{Q}$ affects spectral ratio estimates. Geophysics 80, A39A44.

Gurevich B., Zyrianov V.B. and Lopatnikov S.L. 1997. Seismic attenuation in finely layered porous rocks: effects of fluid flow and scattering. Geophysics 62, 319-324.

Hunziker J., Favino M., Caspari E., Quintal B., Rubino J., Krause R. et al. 2018. Seismic attenuation and stiffness modulus dispersion in porous rocks containing stochastic fracture networks. Journal of Geophysical Research: Solid Earth 123, 125143.

Jänicke R., Quintal B. and Steeb H. 2015. Numerical homogenization of mesoscopic loss in poroelastic media. European Journal of Mechanics A/Solids 49, 382-395.

Johnson D.L., Koplik J. and Dashen R. 1987. Theory of dynamic permeability and tortuosity in fluid-saturated porous media. Journal of Fluid Mechanics 176, 379-402.

Lisitsa V., Podgornova O. and Tcheverda V. 2010. On the interface error analysis for finite difference wave simulation. Computational Geosciences 14, 769-778.

Liu E. and Martinez A. 2012. Seismic Fracture Characterization: Concepts and Practical Applications. European Association of Geoscientists and Engineers.

Main I.G., Peacock S. and Meredith P.G. 1990. Scattering attenuation and the fractal geometry of fracture systems. Pure and Applied Geophysics 133, 283-304.

Mangriotis M.D., Rector J.W., Herkenhoff E.F. and Neu J.C. 2013. Scattering versus intrinsic attenuation in the vadose zone: a VSP experiment. Geophysics 78, B49-B63.

Masson Y.J. and Pride S.R. 2007. Poroelastic finite difference modeling of seismic attenuation and dispersion due to mesoscopicscale heterogeneity. Journal of Geophysical Research 112, B03204.

Masson Y.J. and Pride S.R. 2010. Finite-difference modeling of Biot's poroelastic equations across all frequencies. Geophysics 75 , N33-N41.

Masson Y.J., Pride S.R. and Nihei K.T. 2006. Finite difference modeling of Biot's poroelastic equations at seismic frequencies. Journal of Geophysical Research: Solid Earth 111.

Milani M., Monachesi L., Sabbione J.I., Rubino J. and Holliger K. 2016. A generalized effective anisotropic poroelastic model for periodically layered media accounting for both biot's global and interlayer flows. Geophysical Prospecting 64, 11351148.

Moczo P., Kristek J., Vavrycuk V., Archuleta R.J. and Halada L. 2002. 3D heterogeneous staggered-grid finite-differece modeling of seismic motion with volume harmonic and arithmetic averaging of elastic moduli and densities. Bulletin of the Seismological Society of America 92, 3042-3066.

Müller T.M., Gurevich B. and Lebedev M. 2010. Seismic wave attenuation and dispersion resulting from wave-induced flow in porous rocks - a review. Geophysics 75, 75A147-75A164.

Murai Y. 2007. Scattering attenuation, dispersion and reflection of SH waves in two-dimensional elastic media with densely distributed cracks. Geophysical Journal International 168, 211223.

Novikov M., Caspari E., Holliger K., Quintal B., Lisitsa V., Rubino J.G. et al. 2017a. Numerical study of fracture connectivity response in seismic wavefields. SEG Technical Program, Expanded Abstracts, 3786-3790. Society of Exploration Geophysicists.

Novikov M., Caspari E., Lisitsa V., Quintal B., Rubino G.J. and Holliger K. 2017b. Attenuation in fluid-saturated fractured porous media: quasi-static numerical upscaling and wave propagation modeling. Poromechanics VI, 1499-1506.

Pride S.R. 2005. Relationships between seismic and hydrological properties. In: Hydrogeophysics, Chap. 9 (eds Y. Rubin and S. Hubbard), pp. 253-291. Springer.

Quintal B., Jänicke R., Rubino J.G., Steeb H. and Holliger K. 2014. Sensitivity of S-wave attenuation to the connectivity of fractures in fluid-saturated rocks. Geophysics 79, WB15-WB24.

Quintal B., Steeb H., Frehner M. and Schmalholz M. 2011. Quasistatic finite element modeling of seismic attenuation and dispersion due to wave-induced fluid flow in poroelastic media. Journal of Geophysical Research 116, 1-17.

Rubino J.G., Caspari E., Müller T.M. and Holliger K. 2017. Fracture connectivity can reduce the velocity anisotropy of seismic waves. Geophysical Journal Internationl 210, 223-227.

Rubino J., Caspari E., Müller T.M., Milani M., Barbosa N.D. and Holliger K. 2016. Numerical upscaling in 2-D heterogeneous poroelastic rocks: Anisotropic attenuation and dispersion of seismic waves. Journal of Geophysical Research: Solid Earth 121, 66986721.

Rubino J.G., Guarracino L., Müller T.M. and Holliger K. 2013. Do seismic waves sense fracture connectivity? Geophysical Research Letters 40, 692-696.

Rubino J.G., Müller T.M., Guarracino L., Milani M. and Holliger K. 2014. Seismoacoustic signatures of fracture connectivity. Journal of Geophysical Research 119, 2252-2271.

Rubino J.G., Ravazzoli C.L. and Santos J.E. 2009. Equivalent viscoelastic solids for heterogeneous fluid-saturated porous rocks. Geophysics 74, N1-N13.

Rytov S.M., Kravtsov Y.A. and Tatarskii V.I. 1988. Principles of Statistical Radiophysics 2. Correlation Theory of Random Processes. Springer-Verlag.

Saenger E.H., Krüger O.S. and Shapiro S.A. 2004. Effective elastic properties of randomly fractured soils: 3D numerical experiments. Geophysical Prospecting 52, 183-195.

Samarskii A.A. 2001. The Theory of Difference Schemes, Vol. 240. CRC Press.

Vinci C., Renner J. and Steeb H. 2014. On attenuation of seismic waves associated with flow in fractures. Geophysical Research Letters $41,7515-7523$. 
Virieux J. 1986. P-SV wave propagation in heterogeneous media: velocity-stress finite-difference method. Geophysics 51, 889901.

Vishnevsky D., Lisitsa V., Tcheverda V. and Reshetova G. 2014. Numerical study of the interface errors of finite-difference simulations of seismic waves. Geophysics 79, T219-T232.

Vlastos S., Liu E., Main I.G. and Clement N. 2007. Numerical simulation of wave propagation in 2-D fractured media: scattering attenuation at different stages of the growth of a fracture population. Geophysical Journal International 171, 865880 .

Wenzlau F. and Müller T.M. 2009. Finite-difference modeling of wave propagation and diffusion in poroelastic media. Geophysics 74, T55-T66.

\section{APPENDIX A: FINITE DIFFERENCE APPROXIMATIONS}

For the wave propagation simulation in fluid-saturated porous media, we utilize the standard staggered grid finite difference scheme to approximate the system of equations (3)-(6). Following, Virieux (1986), Masson et al. (2006) and Masson and Pride (2010), a grid with integer and half-integer nodes is introduced; that is $t^{n}=n \tau, t^{n+1 / 2}=(n+1 / 2) \tau, x_{i}=i h_{x}$, $x_{i+1 / 2}=(i+1 / 2) h_{x}, z_{j}=j h_{z}, z_{j+1 / 2}=(j+1 / 2) h_{z}$, where $n$, $i$ and $j$ are integers and $\tau, h_{x}$ and $h_{z}$ are the grid steps along the temporal and spatial directions. We use the second-order centred finite difference operators

$$
\begin{array}{ll}
D_{t}[f]_{i, j}^{n}=\frac{f_{i, j}^{n+1 / 2}-f_{i, j}^{n-1 / 2}}{\tau}, & A_{t}[f]_{i, j}^{n}=\frac{f_{i, j}^{n+1 / 2}+f_{i, j}^{n-1 / 2}}{2}, \\
D_{x}[f]_{i, j}^{n}=\frac{f_{i+\frac{1}{2}, j}^{n}-f_{i-\frac{1}{2}, j}^{n}}{h_{x}}, & D_{z}[f]_{i, j}^{n}=\frac{f_{i, j+\frac{1}{2}}^{n}-f_{i, j-\frac{1}{2}}^{n}}{h_{z}},
\end{array}
$$

where $f_{i, j}^{n}=f\left(t^{n}, x_{i}, z_{j}\right)$ denotes a grid function defined at described points. To construct the finite difference scheme, the wavefield components are defined at different grid nodes (Fig. 14), assuming that the model parameters are constant within each grid cell; that is inside the rectangles $\left[x_{i-1 / 2}, x_{i+1 / 2}\right] \times\left[z_{j-1 / 2}, z_{j+1 / 2}\right]$. The model interfaces are aligned with the grid lines, ensuring the second-order of convergence even for discontinuous coefficients as discussed in, for example Moczo et al. (2002); Lisitsa, Podgornova and Tcheverda (2010); Vishnevsky et al. (2014). To do so, we apply the balance technique or the finite-volume approximation on a rectangular mesh (Samarskii 2001) to equation (3)-(6):

$$
\begin{aligned}
& \left\langle\rho_{f} \frac{T}{\phi}\right\rangle_{i+1 / 2, j} D_{t}\left[q_{x}\right]_{i+1 / 2, j}^{n+1 / 2}+\left\langle\rho_{f}\right\rangle_{i+1 / 2, j} D_{t}\left[u_{x}\right]_{i+1 / 2, j}^{n+1 / 2} \\
& \quad+\left\langle\frac{\eta}{k_{0}}\right\rangle_{i+1 / 2, j} A_{t}\left[q_{x}\right]_{i+1 / 2, j}^{n+1 / 2}=-D_{x}[p]_{i+1 / 2}^{n+1 / 2},
\end{aligned}
$$

$$
\begin{aligned}
& \left\langle\rho_{f} \frac{T}{\phi}\right\rangle_{i, j+1 / 2} D_{t}\left[q_{z}\right]_{i, j+1 / 2}^{n+1 / 2}+\left\langle\rho_{f}\right\rangle_{i, j+1 / 2} D_{t}\left[u_{z}\right]_{i, j+1 / 2}^{n+1 / 2} \\
& +\left\langle\frac{\eta}{k_{0}}\right\rangle_{i, j+1 / 2} A_{t}\left[q_{x}\right]_{i, j+1 / 2}^{n+1 / 2}=-D_{z}[p]_{i, j+1 / 2}^{n+1 / 2},
\end{aligned}
$$$$
\left\langle\rho_{f}\right\rangle_{i+1 / 2, j} D_{t}\left[q_{x}\right]_{i+1 / 2, j}^{n+1 / 2}+\langle\rho\rangle_{i+1 / 2, j} D_{t}\left[u_{x}\right]_{i+1 / 2, j}^{n+1 / 2}
$$$$
=D_{x}\left[\sigma_{x x}\right]_{i+1 / 2, j}^{n+1 / 2}+D_{z}\left[\sigma_{x z}\right]_{i+1 / 2, j}^{n+1 / 2} \text {, }
$$$$
\left\langle\rho_{f}\right\rangle_{i, j+1 / 2} D_{t}\left[q_{z}\right]_{i, j+1 / 2}^{n+1 / 2}+\langle\rho\rangle_{i, j+1 / 2} D_{t}\left[u_{z}\right]_{i, j+1 / 2}^{n+1 / 2}
$$$$
=D_{x}\left[\sigma_{x z}\right]_{i, j+1 / 2}^{n+1 / 2}+D_{z}\left[\sigma_{z z}\right]_{i, j+1 / 2}^{n+1 / 2} \text {, }
$$

$$
\begin{aligned}
& D_{t}\left[\sigma_{x x}\right]_{i, j}^{n}=(\lambda+2 \mu)_{i, j} D_{x}\left[u_{x}\right]_{i, j}^{n}+(\lambda)_{i, j} D_{z}\left[u_{z}\right]_{i, j}^{n} \\
& \quad+(\alpha M)_{i, j}\left(D_{x}\left[q_{x}\right]_{i, j}^{n}+D_{z}\left[q_{z}\right]_{i, j}^{n}\right), \\
& D_{t}\left[\sigma_{z z}\right]_{i, j}^{n}=(\lambda)_{i, j} D_{x}\left[u_{x}\right]_{i, j}^{n}+(\lambda+2 \mu)_{i, j} D_{z}\left[u_{z}\right]_{i, j}^{n} \\
& \quad+(\alpha M)_{i, j}\left(D_{x}\left[q_{x}\right]_{i, j}^{n}+D_{z}\left[q_{z}\right]_{i, j}^{n}\right),
\end{aligned}
$$

$$
\begin{aligned}
& D_{t}\left[\sigma_{x z}\right]_{i+1 / 2, j+1 / 2}^{n} \\
& \quad=\{\mu\}_{i+1 / 2, j+1 / 2}\left(D_{z}\left[u_{x}\right]_{i+1 / 2, j+1 / 2}^{n}+D_{x}\left[u_{z}\right]_{i+1 / 2, j+1 / 2}^{n}\right),
\end{aligned}
$$

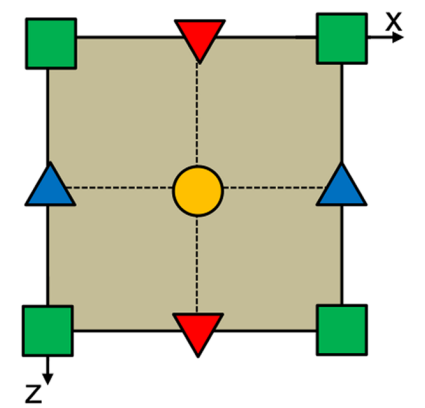

Figure 14 Schematic illustration of a staggered finite difference grid cell with the associated wave field components and material properties. 


$$
\begin{aligned}
D_{t}[p]_{i, j}^{n}= & -(\alpha M)_{i, j}\left(D_{x}\left[v_{x}\right]_{i, j}^{n}+D_{z}\left[v_{z}\right]_{i, j}^{n}\right) \\
& +(M)_{i, j}\left(D_{x}\left[q_{x}\right]_{i, j}^{n}+D_{z}\left[q_{z}\right]_{i, j}^{n}\right),
\end{aligned}
$$

where for any grid function $f_{i, j}$

$$
\begin{aligned}
\langle f\rangle_{i+1 / 2, j} & =0.5\left(f_{i, j}+f_{i+1, j}\right), \\
\langle f\rangle_{i, j+1 / 2} & =0.5\left(f_{i, j}+f_{i, j+1}\right), \\
\{f\}_{i+1 / 2, j+1 / 2} & =4\left(\frac{1}{f_{i, j}}+\frac{1}{f_{i+1, j}}+\frac{1}{f_{i, j+1}}+\frac{1}{f_{i+1, j+1}}\right)^{-1} .
\end{aligned}
$$

Following Masson et al. (2006), the Courant stability condition for the considered scheme is

$\tau V_{p}^{\max } \sqrt{\frac{1}{h_{x}^{2}}+\frac{1}{h_{z}^{2}}} \leq 1$,

where $V_{p}^{\max }=\max _{\chi, \omega} \Re\left(V_{p}\right), V_{p}$ is fast P-wave phase velocity, and $\chi=\eta / k_{0}$. Note that in this study, we use the secondorder approximation in space. The approximation can be easily improved by using higher order operators. However, we consider the case where heterogeneities, and thus the grid steps, are much smaller than the wavelength, thus the numerical dispersion is negligible even for the low-order approximations. Moreover, the formal increase of the order of the approximation does not improve the convergence rate of the solution in inhomogeneous media (Vishnevsky et al. 2014).

\section{APPENDIX B: SPECTRAL RATIO METHOD - PHASE VELOCITY AND QUALITY FACTOR}

Let us consider a plane wave propagating in $z$-direction through the fractured medium

$v(t, z)=v_{0} \mathrm{e}^{i \omega(t-z / c(\omega))}$,

where $c(\omega)$ is the complex frequency-dependent phase velocity of the medium. The plane wave amplitude $v_{0}$ in the transmission experiment depends on the attenuation in the fractured domain and on the transmission losses across the fractured domain, but remains constant in the homogeneous medium. Let $z=z_{1}$ and $z=z_{2}=z_{1}+L$ be the two receiver positions. Then the average of the signals recorded at the two receiver positions is given by

$$
\begin{aligned}
\left\langle v\left(t, x, z_{1}\right)\right\rangle_{x} & =v\left(t, z_{1}\right)=v_{0} \mathrm{e}^{i \omega\left(t-z_{1} / c(\omega)\right)}, \\
\left\langle v\left(t, x, z_{2}\right)\right\rangle_{x} & =v\left(t, z_{2}\right)=v_{0} \mathrm{e}^{i \omega\left(t-\left(z_{1}+L\right) / c(\omega)\right)} \\
& =v\left(t, z_{1}\right) \mathrm{e}^{-i \omega L / c(\omega)}
\end{aligned}
$$

The same relations hold in the frequency domain and equation B3 can be written as

$v\left(\omega, z_{2}\right)=v\left(\omega, z_{1}\right) \mathrm{e}^{-i \omega L \Re(s)} \mathrm{e}^{\omega L \Im(s)}$,

where $s(\omega)$ is the inverse of the complex valued phase velocity. From this, one can easily obtain the imaginary part of $s(\omega)$ as follows:

$\Im(s)=\frac{1}{\omega L} \log \frac{\left|v\left(\omega, z_{2}\right)\right|}{\left|v\left(\omega, z_{1}\right)\right|}$.

The real part of $s(\omega)$ is the argument of the ratio $\left|v\left(\omega, z_{2}\right)\right| /\left|v\left(\omega, z_{1}\right)\right|$ and thus is not single-valued. To determine the phase uniquely, we pick the travel times $t_{1}$ and $t_{2}$ at the maximum amplitude of the two signals for the two receiver positions, which provides an approximation for the phase velocity

$V_{p}^{0}=\frac{L}{t_{2}-t_{1}}$.

Then, the phase is chosen so that the following condition is fulfilled

$\left|\frac{\mathfrak{R}(s(\omega))}{\omega L}-\frac{\omega L}{V_{p}^{0}}\right|<\pi$.

From the resulting real and imaginary parts of the complexvalued slowness, the real-valued phase velocity and the quality factor can be reconstructed as

$v_{p}=\frac{1}{\Re(s(\omega))}$,

$Q_{p}^{-1}=\frac{-\Im\left(s^{2}(\omega)\right)}{\Re\left(s^{2}(\omega)\right)}$.

\section{APPENDIX C: BOUNDARY CONDITIONS OF THE OSCILLATORY TESTS}

To estimate the frequency-dependent stiffness coefficients, we solve Biot's (1941) quasi-static poroelastic equations (11)(13) for the following displacement boundary conditions. The computational domain is given by $\Omega=\left[0, L_{x}\right] \times\left[0, L_{z}\right]$ with the boundary $\Gamma=\Gamma^{L} \cup \Gamma^{B} \cup \Gamma^{R} \cup \Gamma^{T}$, where

$\Gamma^{L}=\{(x, z) \in \Omega: x=0\}$,

$\Gamma^{R}=\left\{(x, z) \in \Omega: x=L_{x}\right\}$,

$\Gamma^{B}=\{(x, z) \in \Omega: z=0\}$,

$\Gamma^{T}=\left\{(x, z) \in \Omega: z=L_{z}\right\}$. 
The boundary conditions for the two compressional tests are given by

$$
\begin{aligned}
& \boldsymbol{u} \cdot \boldsymbol{v}=-\Delta u, \quad(x, z) \in \Gamma^{T} \cup \Gamma^{B}, \\
& \boldsymbol{u} \cdot \boldsymbol{v}=0, \quad(x, z) \in \Gamma^{L} \cup \Gamma^{R}(\text { Test } 1),
\end{aligned}
$$

$\boldsymbol{u} \cdot \boldsymbol{v}=-\Delta u, \quad(x, z) \in \Gamma^{R} \cup \Gamma^{L}$,

$\boldsymbol{u} \cdot \boldsymbol{v}=0, \quad(x, z) \in \Gamma^{T} \cup \Gamma^{B}($ Test 2$)$,

$$
\boldsymbol{w} \cdot \boldsymbol{v}=0, \quad(x, z) \in \Gamma, \quad(\boldsymbol{\sigma} \boldsymbol{v}) \cdot \chi=0, \quad(x, z) \in \Gamma,
$$

where $\boldsymbol{v}$ and $\chi$ denote the unit outer normal and unit tangent on $\Gamma$ so that $\{\boldsymbol{v}, \chi\}$ is an orthonormal system on $\Gamma$. The third test is a simple shear test with the following boundary conditions

$$
\begin{aligned}
& u \cdot \chi=\Delta u, \quad(x, z) \in \Gamma^{T} \cup \Gamma^{B}, \\
& \boldsymbol{u} \cdot \chi=0, \quad(x, z) \in \Gamma^{L} \cup \Gamma^{R}, \\
& \boldsymbol{w} \cdot \boldsymbol{v}=0, \quad(x, z) \in \Gamma, \quad(\sigma \boldsymbol{v}) \cdot \chi=0, \quad(x, z) \in \Gamma .
\end{aligned}
$$

\section{APPENDIX D: TRANSMISION LOSSES - PLANE WAVE AMPLITUDE ANALYSIS}

To estimate transmission losses, we perform a $2 \mathrm{D}$ plane wave amplitude analysis for an anisotropic viscoelastic layer $\Omega_{M}$ embedded in an isotropic elastic medium $\left(\Omega_{U}\right.$ and $\left.\Omega_{L}\right)$. For a general anisotropic case under plane strain conditions, the properties of a $2 \mathrm{D}$ viscoelastic layer can be obtained from the oscillatory test (Rubino et al. 2016). The constitutive relations in the domain $\Omega_{M}$ are given by:

$\sigma_{11}=C_{11} \epsilon_{11}+C_{12} \epsilon_{22}+2 C_{16} \epsilon_{12}$,
$\sigma_{22}=C_{12} \epsilon_{11}+C_{22} \epsilon_{22}+2 C_{26} \epsilon_{12}$,

$\sigma_{12}=C_{16} \epsilon_{11}+C_{26} \epsilon_{22}+2 C_{66} \epsilon_{12}$,

where the stress $\sigma_{i j}$, the strain, $\epsilon_{i j}$, and the coefficients $C_{i j}$, are complex-valued and frequency-dependent. The isotropic constitutive relations in the upper $\Omega_{U}$ and lower domain $\Omega_{L}$ are

$\sigma_{i j}=2 \mu \epsilon_{i j}+\delta_{i j} \lambda \nabla u$

where $\mu, \lambda$ and $u$ are the shear modulus, Lamé parameter and displacement, respectively. For plane waves the expressions of the potentials in each layer are known (Dutta and Odé 1983; Barbosa et al. 2016). Further, assuming the following set of conditions at the upper $\Gamma_{1}$ and lower boundary $\Gamma_{2}$ of the viscoelastic layer

$u_{1}^{\Omega_{U}}=u_{1}^{\Omega_{M}}$ in $\Gamma_{1}$ and $u_{1}^{\Omega_{M}}=u_{1}^{\Omega_{L}}$ in $\Gamma_{2}$

$u_{2}^{\Omega_{U}}=u_{2}^{\Omega_{M}}$ in $\Gamma_{1}$ and $u_{2}^{\Omega_{M}}=u_{2}^{\Omega_{L}}$ in $\Gamma_{2}$

$\sigma_{12}^{\Omega_{U}}=\sigma_{12}^{\Omega_{M}}$ in $\Gamma_{1}$ and $\sigma_{12}^{\Omega_{M}}=\sigma_{12}^{\Omega_{L}}$ in $\Gamma_{2}$

$\sigma_{22}^{\Omega_{U}}=\sigma_{22}^{\Omega_{M}}$ in $\Gamma_{1}$ and $\sigma_{22}^{\Omega_{M}}=\sigma_{22}^{\Omega_{L}}$ in $\Gamma_{2}$

we can construct a linear system of equations for the potential amplitudes. Let us denote a wave vector $\mathbf{k}=(m, l)$, where the horizontal $m$ and vertical component $l$ are given by

$m=k_{p}^{U} \sin \left(\theta_{i}\right)$

$l_{n}^{r}=\sqrt{\left(k_{n}^{r}\right)^{2}-m^{2}} \quad n=P, S$ and $r=\Omega_{U}, \Omega_{M}, \Omega_{L}$.

The horizontal component is constant for all wave modes, whereas the vertical component depends on the wave type denoted here by $P$ and $S$ for the P- and S-waves, respectively. Assuming that the incident wave is homogeneous with 
an incident angle $\theta_{i}$, the linear system can be written as follows:

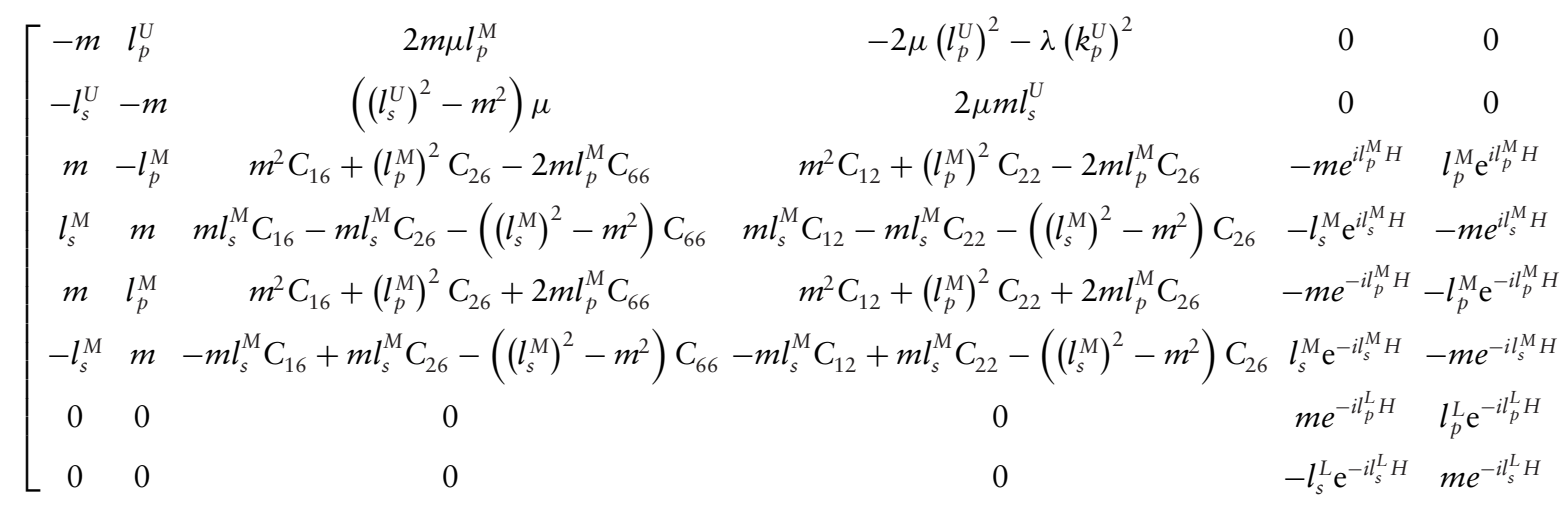

$$
\left.\begin{array}{cc}
0 & 0 \\
0 & 0 \\
\left.\left(-m^{2} C_{16}-l_{p}^{M}\right)^{2} C_{26}+2 m l_{p}^{M} C_{66}\right) \mathrm{e}^{i l_{p}^{M} H} & \left.\left(-m^{2} C_{12}-l_{p}^{M}\right)^{2} C_{22}+2 m l_{p}^{M} C_{26}\right) \mathrm{e}^{i l_{p}^{M} H} \\
\left(-m l_{s}^{M} C_{16}+m l_{s}^{M} C_{26}+\left(\left(l_{s}^{M}\right)^{2}-m^{2}\right) C_{66}\right) \mathrm{e}^{i l l_{s}^{M} H} & \left(-m l_{s}^{M} C_{12}+m l_{s}^{M} C_{22}+\left(\left(l_{s}^{M}\right)^{2}-m^{2}\right) C_{26}\right) \mathrm{e}^{i l_{s}^{M} H} \\
\left(-m^{2} C_{16}-\left(l_{p}^{M}\right)^{2} C_{26}-2 m l_{p}^{M} C_{66}\right) \mathrm{e}^{-i l_{p}^{M} H} & \left(-m^{2} C_{12}-\left(l_{p}^{M}\right)^{2} C_{22}-2 m l_{p}^{M} C_{26}\right) \mathrm{e}^{-i l_{p}^{M} H} \\
\left(m l_{s}^{M} C_{16}-m l_{s}^{M} C_{26}+\left(\left(l_{s}^{M}\right)^{2}-m^{2}\right) C_{66}\right) \mathrm{e}^{-i l_{s}^{M} H} & \left(m l_{s}^{M} C_{12}-m l_{s}^{M} C_{22}+\left(\left(l_{s}^{M}\right)^{2}-m^{2}\right) C_{26}\right) \mathrm{e}^{-i l_{s}^{M} H} \\
2 m l_{p}^{L} \mu \mathrm{e}^{-i l_{p}^{L} H} & \left(2 \mu\left(l_{p}^{L}\right)^{2}+\lambda k_{p}^{2}\right) \mathrm{e}^{-i l_{p}^{L} H} \\
-\left(\left(l_{s}^{M}\right)^{2}-m^{2}\right) \mu \mathrm{e}^{-i l l_{s}^{L} H} & 2 m l_{s}^{L} \mu \mathrm{e}^{-i l_{s}^{L} H}
\end{array}\right]
$$

$$
\left[\begin{array}{c}
A_{r} \\
B_{r} \\
A_{u} \\
B_{u} \\
A_{d} \\
B_{d} \\
A_{t} \\
B_{t}
\end{array}\right]=\left[\begin{array}{c}
m \\
l_{p}^{U} \\
2 m \mu l_{p}^{U} \\
2 \mu\left(l_{p}^{U}\right)^{2}+\lambda k_{p}^{2} \\
0 \\
0 \\
0 \\
0
\end{array}\right]
$$

where $A_{r}, B_{r}, A_{u}, B_{u}, A_{d}, B_{d}, A_{t}, B_{t}$ are the potential amplitudes of the reflected, the up-going, the down-going and the transmitted $\mathrm{P}$ - and $\mathrm{S}$-waves. Here, we only retrieve the amplitudes for an incidence angle of $\theta_{i}=0$, since this corresponds to the set-up of the transmission experiment. Once we have solved the linear system for these potential amplitudes, we can compute the solid displacement field of the incident $u^{i}$ and transmitted wave $u^{T}$

$$
\begin{aligned}
& u^{i}\left(y_{1}\right)=-i l_{p} \mathrm{e}^{-i l_{p} y_{1}} A_{i}, \\
& u^{t}\left(y_{2}\right)=-i l_{p} \mathrm{e}^{-i l_{p} y_{2}} A_{t},
\end{aligned}
$$


where the incident amplitude $A_{i}$ is assumed to be 1 . Assuming that the decay of the amplitude is caused by the viscoelastic layer, we can alternatively express the two displacement fields similar to Appendix B in terms of an effective wavenumber $\left(l_{e f f}\right)$

$u^{i}\left(y_{1}\right)=-i l_{p}^{e f f} \mathrm{e}^{-i l_{p}^{e f f} y_{1}}$

$u^{t}\left(y_{2}\right)=-i l_{p}^{\text {eff }} \mathrm{e}^{-i l_{p}^{\text {eff }} y_{2}}$

where $l_{p}$ is the $\mathrm{P}$-wave number of the embedding homogeneous background. Combing these two sets of expressions leads to the relation for the effective P-wavenumber

$l_{p}^{e f f}=\frac{-i l_{p} L+\ln \left(A_{t}\right)}{-i L}$,

where $L=y_{2}+y_{1}$ and $y_{2}$ and $y_{1}$ are the positions of the second and first receiver line, respectively. This effective P-wave number describes the three-layer case. By replacing in equation (D13) $y_{1}$ and $y_{2}$ with the interfaces of the viscoelastic layer leads to the effective wavenumber of the fractured medium

$l_{p}^{e f f}=\frac{-i l_{p} L_{f r a c}+\ln \left(A_{t}\right)}{-i L}$.

\section{APPENDIX E: ESTIMATION OF ATTENUATION DUE TO SCATTERING}

To estimate the energy dissipation caused by scattering for a system of randomly distributed heterogeneities in a
2D acoustic medium, we compute the effective scattering cross-section after Rytov et al. (1988):

$\sigma(\phi)=\frac{1}{2} k^{3} \pi \Phi_{S}(Q(\phi)) \quad$ with $Q(\phi)=k\left(n_{s}(\phi)-n\right)$,

where $k=\omega /\langle v\rangle$ is the modulus of the wave vector and $\boldsymbol{n}_{\boldsymbol{s}}(\phi)$ the vector that defines the direction of the scattered waves. The vector $\boldsymbol{n}$ defines the direction of the incident plane wave. The spectral density function $\Phi_{S}(Q(\phi))$ is the frequency counterpart of the correlation function of the slowness fluctuations $\tilde{S}=\left(V_{p}(\mathbf{r})\right)^{-1}-\left\langle\left(V_{p}(\mathbf{r})\right)^{-1}\right\rangle$ of the fractured domain. $V_{p}$ is the $\mathrm{P}$-wave phase velocity. As we consider a uniform distribution of fractures, the slowness fluctuation field is statistically quasi-homogeneous. Hence, its correlation function can be represented as

$\psi_{S}\left(\boldsymbol{r}_{1}, \boldsymbol{r}_{2}\right)=\left\langle\tilde{S}\left(\boldsymbol{r}_{1}\right) \tilde{S}\left(\boldsymbol{r}_{2}\right)\right\rangle=\psi_{S}(\rho, R)$,

where $\rho=\mathbf{r}_{1}-\mathbf{r}_{2}, \quad R=\left(\mathbf{r}_{1}+\mathbf{r}_{2}\right) / 2$, and $\psi_{S}(\rho, R)$ slowly vary with $\boldsymbol{R}$. Thus, the spectral density can be obtained from

$\Phi_{S}(\kappa, R)=\frac{1}{(2 \pi)^{2}} \int \psi_{S}(\rho, R) \mathrm{e}^{-i \kappa \rho} d \rho$,

and the scattering attenuation is given by

$\frac{1}{Q(\omega)}=\frac{\left\langle V_{p}(\boldsymbol{r})\right\rangle \int_{0}^{2 \pi} \sigma(\phi) d \phi}{\omega \pi}$,

where $\left\langle V_{p}(\boldsymbol{r})\right\rangle$ is the average P-wave phase velocity. 